\title{
Natural neutrino sector in a 331-model with Froggatt-Nielsen mechanism
}

\author{
Katri Huitu, Niko Koivunen and Timo J. Kärkkäinen \\ Department of Physics, and Helsinki Institute of Physics, \\ P.O. Box 64, FI-00014 University of Helsinki, Finland \\ E-mail: katri.huitu@helsinki.fi, niko.koivunen@helsinki.fi, \\ karkkainen@caesar.elte.hu
}

Abstract: The extensions of the Standard Model based on the $\mathrm{SU}(3)_{c} \times \mathrm{SU}(3)_{L} \times \mathrm{U}(1)_{X}$ gauge group (331-models) have been advocated to explain the number of fermion families in nature. It has been recently shown that the Froggatt-Nielsen mechanism, a popular way to explain the mass hierarchy of the charged fermions, can be incorporated into the 331-setting in an economical fashion (FN331). In this work we extend the FN331-model to include three right-handed neutrino singlets. We show that the seesaw mechanism is realized in this model. The scale of the seesaw mechanism is near the $\mathrm{SU}(3)_{L} \times \mathrm{U}(1)_{X}$-breaking scale. The model we present here simultaneously explains the mass hierarchy of all the fermions, including neutrinos, and the number of families.

Keywords: Beyond Standard Model, Global Symmetries, Neutrino Physics, Spontaneous Symmetry Breaking

ARXIV EPRINT: 1908.09384 


\section{Contents}

1 Introduction 1

2 Particle content 3

2.1 Fermion representations 3

2.2 Scalar sector 4

2.3 Gauge sector 6

$\begin{array}{lll}\text { 2.3.1 Charged gauge bosons } & 6\end{array}$

3 The Yukawa sector and the Froggatt-Nielsen mechanism in the 331$\begin{array}{ll}\text { framework } & 7\end{array}$

4 Charged lepton Yukawa couplings and masses $\quad 9$

5 Neutrino mass matrix $\quad 9$

6 Neutrino masses and eigenstates $\quad 11$

$\begin{array}{lll}6.1 & \text { Neutrino mass matrices } & 12\end{array}$

$\begin{array}{lll}6.2 & \text { Neutrino eigenstates } & 14\end{array}$

$\begin{array}{lll}7 & \text { Neutrino coupling to charged gauge bosons and PMNS-matrix } & 14\end{array}$

8 Constraints and numerical examples $\quad 16$

$\begin{array}{ll}\text { 8.1 The FN-charges for the numerical example } & 17\end{array}$

$\begin{array}{lll}8.2 & \text { Numerical values for leptons } & 18\end{array}$

9 Conclusion $\quad 20$

A Scalar mass matrices $\quad 22$

A.1 CP-even scalars 22

A.2 CP-odd scalars 22

$\begin{array}{lll}\text { A.3 Charged scalars } & 22\end{array}$

B Neutral gauge boson masses $\quad 22$

\section{Introduction}

After the discovery of the Higgs boson at the Large Hadron Collider, the last elementary particle predicted by the Standard Model (SM) has been confirmed. Today, particle physics has moved on to a new era, where we attempt to answer the problems plagueing the SM 
with economical extensions. The problems include number of generations, nonzero neutrino mass, neutrino mixing and fermion mass hierarchies.

In Nature three generations of quarks and leptons have been observed. Number of neutrino flavours is $2.984 \pm 0.008$ [1]-[7], which is a statistical fit to SM using LEP data. This is a strong indication for exactly three generations of matter, which, however, is not imposed by SM itself. We know from neutrino oscillation experiments that at least two of the three SM neutrinos are massive, with masses less than $0.1 \mathrm{eV}$ and the sum of their masses is less than $0.12 \mathrm{eV}$ from cosmological constraints by the PLANCK experiment. Neutrino masses are not included in the Standard Model, and they are six orders of magnitude lighter than the next lightest massive particle, electron, and twelve orders of magnitudes lighter than the heaviest particle, top quark. This huge range of different masses gives birth to the flavour problem.

Extensions of the Standard Model based on the $\mathrm{SU}(3)_{c} \times \mathrm{SU}(3)_{L} \times \mathrm{U}(1)_{X}$ gauge group (331-models) have been proposed in the literature to explain the number of fermion families in Nature. In the traditional 331-models [8]-[22] the gauge anomalies cancel only if the number of fermion familes is three. The $\mathrm{SU}(3)_{c} \times \mathrm{SU}(3)_{L} \times \mathrm{U}(1)_{X}$ gauge group contains one additional diagonal generator compared to the SM-gauge group $\mathrm{SU}(3)_{c} \times \mathrm{SU}(2)_{L} \times$ $\mathrm{U}(1)_{Y}$. This means that the electric charge can be defined in multiple different ways in the 331-models:

$$
Q=T_{3}+\beta T_{8}+X,
$$

where the parameter $\beta$ defines the particle content of the model. The models with $\beta=$

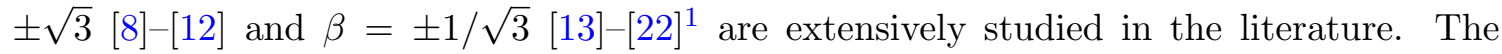
models with $\beta= \pm \sqrt{3}$ contain particles with exotic electric charges such as doubly charged scalars and gauge bosons. They also contain a very large scalar sector, composed of three $\mathrm{SU}(3)_{L}$-triplets and an $\mathrm{SU}(3)_{L}$-sextet. The models based on the $\beta= \pm 1 / \sqrt{3}$ on the other hand have simpler scalar sector, composed from only three $\mathrm{SU}(3)_{L}$-triplets. The models based on $\beta= \pm 1 / \sqrt{3}$ do not contain particles with exotic electric charges. Also the models with $\beta=0$ have been studied [23].

Even though the 331-models can shed light on the number of fermion familes, the fermion mass hierarchy is left unexplained in the traditional models. Recently it was shown that the Froggatt-Nielsen mechanism [24] can be incorporated into the 331-models with $\beta= \pm 1 \sqrt{3}$ without extending the scalar sector $[25,26]$. The Froggatt-Nielsen mechanism (FN) is a well established method to explain the mass hierarchy of the fermions, and a 331model with incorporated FN-mechanism (FN331) can therefore simultaneously explain both the number of fermion families and the mass hierarchy of the charged fermions. The neutrino masses and mixings are not naturally explained in FN331-model, however. The neutrino mass matrix is antisymmetric in the FN331-model and therefore one of the neutrinos is massless and the two other mass degenerate at tree-level. Loop corrections are needed to lift the one eigenvalue from zero and to break the degeneracy of the other two [15]. This neutrino sector is identical to the one presented in [14]-[22].

\footnotetext{
${ }^{1}$ The model presented in [13] does not exhibit the cancellation of gauge anomalies and does not explain the number of fermion families.
} 
Our aim is to extend the neutrino sector to make it natural and explain the neutrino masses and mixings without fine-tuning at tree-level. We propose an extension of the FN331-model where we add three right-handed neutrino singlets to the model. This allows the tree-level masses for all the neutrinos and implementation of seesaw mechanism [29][34] for the neutrino sector. Here the seesaw is combined with the FN-mechanism, which allows the seesaw scale to be low thanks to suppression in the neutrino Yukawa couplings due to the FN-mechanism. The seesaw scale is essentially the same as the $\mathrm{SU}(3)_{L} \times \mathrm{U}(1)_{X^{-}}$ breaking scale. We study as low $\mathrm{SU}(3)_{L} \times \mathrm{U}(1)_{X}$-breaking scale as possible. The suppression of flavour changing neutral currents $(\mathrm{FCNC})$ allows for $\mathrm{SU}(3)_{L} \times \mathrm{U}(1)_{X}$-breaking scale as low as $5 \mathrm{TeV}$ as shown in [26]. The collider bounds on the $Z^{\prime}$ mass however suggest that the $\mathrm{SU}(3)_{L} \times \mathrm{U}(1)_{X}$-breaking scale has to be at least around $7 \mathrm{TeV}$ [27]. We aim to generate singlet neutrinos at medium-energy scale and sterile neutrinos at $\mathrm{TeV}$ scale, utilizing seesaw mechanism. By medium-energy we refer to a mass scale between the active neutrinos and mass of electron. We will present the lowest possible $\mathrm{SU}(3)_{L} \times \mathrm{U}(1)_{X}$ breaking scale consistent with experimental data, which turns out to be approximately $7 \mathrm{TeV}$.

The hierachical structure of the neutrino Yukawa couplings is determined by the FNmechanism. The hierarchy of the neutrino Yukawa couplings can be arranged so that the neutrino mixings are generated without fine-tuning. All the PMNS-matrix elements are experimentally known to be order-one-numbers. This kind of pattern of mixing can be achieved in FN-setting by assigning all the FN-charges of the left-handed leptons to be equal. We show that the correct sub-eV neutrino masses, mass square differences and mixing angles are produced within experimental limits.

The paper is structured as follows. We present the particle content of the model in section 2. In section 3 we review the Froggatt-Nielsen mechanism in the 331-setting, the FN331-model. In sections 4 to 7 we study the lepton mass matrices and mixings and finally in the section 8 we present numerical example for the neutrino masses and mixings.

\section{Particle content}

We propose a model where the gauge group of the Standard Model is extended to $\mathrm{SU}(3)_{C} \times$ $\mathrm{SU}(3)_{L} \times \mathrm{U}(1)_{X}$. We define the electric charge as: ${ }^{2}$

$$
Q=T_{3}-\frac{1}{\sqrt{3}} T_{8}+X,
$$

where the $T_{3}$ and $T_{8}$ are the diagonal $\mathrm{SU}(3)_{L}$ generators. We also introduce global $\mathrm{U}(1)_{F N^{-}}$ symmetry, under which fermions and some of the scalars are charged.

\section{$2.1 \quad$ Fermion representations}

Let us now write down the fermion representations. The left-handed leptons are assigned to $\mathrm{SU}(3)_{L}$-triplets and the right-handed leptons are assigned to $\mathrm{SU}(3)_{L}$-singlets:

$$
L_{L, i}=\left(\begin{array}{c}
\nu_{i} \\
e_{i} \\
\nu_{i}^{\prime}
\end{array}\right)_{L} \sim\left(1,3,-\frac{1}{3}\right), \quad i=1,2,3, \quad e_{R, i} \sim(1,1,-1), \quad N_{R, i} \sim(1,1,0) .
$$

\footnotetext{
${ }^{2}$ The choice $\beta=+\frac{1}{\sqrt{3}}$ would result in essentially a same model.
} 
The numbers in the parantheses label the transformation propeties under the gauge group $\mathrm{SU}(3)_{c} \times \mathrm{SU}(3)_{L} \times \mathrm{U}(1)_{X}$. The $\nu_{L, i}^{\prime}$ and $N_{R, i}$ are new leptons with electric charges 0 . The three right-handed neutrinos $N_{R, i}$ are not present in the model studied in [25, 26] and they allow the tree-level masses for all the neutrinos.

The cancellation of anomalies requires the number of fermion triplets to be the same as antitriplets. This is achieved by assigning two quark families to $\mathrm{SU}(3)_{L}$ antitriplets and one family to a triplet. We choose to assign first quark generations into triplet and the second and the third into an antitriplet:

$$
\begin{aligned}
& Q_{L, 1}=\left(\begin{array}{l}
u_{1} \\
d_{1} \\
U
\end{array}\right)_{L} \sim\left(3,3, \frac{1}{3}\right), \\
& Q_{L, 2}=\left(\begin{array}{c}
d_{2} \\
-u_{2} \\
D_{1}
\end{array}\right)_{L}, \quad Q_{L, 3}=\left(\begin{array}{c}
d_{3} \\
-u_{3} \\
D_{2}
\end{array}\right)_{L} \sim\left(3,3^{*}, 0\right), \\
& u_{R, i} \sim\left(3,1, \frac{2}{3}\right), \quad U_{R} \sim\left(3,1, \frac{2}{3}\right), \\
& d_{R, i} \sim\left(3,1,-\frac{1}{3}\right), \quad D_{R, 1} \sim\left(3,1,-\frac{1}{3}\right), \quad D_{R, 2} \sim\left(3,1,-\frac{1}{3}\right), \quad i=1,2,3 .
\end{aligned}
$$

We have introduced new quarks $D_{1}$ and $D_{2}$ with electric charge $-1 / 3$ and $U$ with electric charge $2 / 3$, which will mix with the SM quarks. All the fermions are also charged under the global Froggatt-Nielsen $\mathrm{U}(1)_{F N}$ symmetry. This will be discussed in detail in the dedicated section 3 .

When we take into account the colour, there are six fermion triplets and six antriplets, ensuring the cancellation of pure $\mathrm{SU}(3)_{L}$-anomaly. All the gauge anomalies will cancel with this particle content. The anomaly cancellation forces one quark generation to be placed in a different representation than the other two. The unequal treatment of quark generations inevitably leads to scalar mediated FCNCs at tree-level, which is a feature plaguing the traditional 331-models [8]-[22]. It was however recently shown that tree-level scalar mediated FCNCs of quarks are suppressed in the FN331-model [26]. This is in contrast to the traditional 331-models, which offer no natural suppression mechanism for the tree-level scalar mediated FCNCs.

\subsection{Scalar sector}

The 331-models with $\beta=-\frac{1}{\sqrt{3}}$ contain only two types of scalar triplets with neutral entries: $X=2 / 3$ and $X=-1 / 3$. One must include at least two $X=-1 / 3$ triplets with $X=-1 / 3$ and one triplet with $X=2 / 3$ in order to generate the masses for all the charged fermions at tree level. We choose to have this minimal scalar sector:

$$
\eta=\left(\begin{array}{c}
\eta^{+} \\
\eta^{0} \\
\eta^{\prime+}
\end{array}\right) \sim\left(1,3, \frac{2}{3}\right), \quad \rho=\left(\begin{array}{c}
\rho^{0} \\
\rho^{-} \\
\rho^{\prime 0}
\end{array}\right) \sim\left(1,3,-\frac{1}{3}\right), \quad \chi=\left(\begin{array}{c}
\chi^{0} \\
\chi^{-} \\
\chi^{\prime 0}
\end{array}\right) \sim\left(1,3,-\frac{1}{3}\right) .
$$




\begin{tabular}{|c|c|c|c|}
\hline Particle & $\eta$ & $\rho$ & $\chi$ \\
\hline FN-charge & -1 & 1 & 0 \\
\hline
\end{tabular}

Table 1. The FN U(1) charges of the scalars.

All the neutral fields can in general develop a nonzero vacuum expectation value (VEV). The minima are related to each other by $\mathrm{SU}(3)_{L}$ rotation. We choose to rotate one of the $X=-1 / 3$ triplet VEVs so that the upper component VEV goes to zero. This rotation will leave the rest of the VEVs general. So we have vacuum structure:

$$
\langle\eta\rangle=\frac{1}{\sqrt{2}}\left(\begin{array}{c}
0 \\
v^{\prime} \\
0
\end{array}\right), \quad\langle\rho\rangle=\frac{1}{\sqrt{2}}\left(\begin{array}{c}
v_{1} \\
0 \\
v_{2}
\end{array}\right), \quad\langle\chi\rangle=\frac{1}{\sqrt{2}}\left(\begin{array}{c}
0 \\
0 \\
u
\end{array}\right) .
$$

The VEVs $v_{2}$ and $u$ break the $\mathrm{SU}(3)_{L}$-symmetry, and we assume them to be: $v_{2}, u \gtrsim$ $\mathcal{O}(\mathrm{TeV})$. The VEVs $v^{\prime}$ and $v_{1}$ break the $\mathrm{SU}(2)_{L}$ symmetry and we take them to be of the order of the electroweak scale.

The scalar triplets in eq. (2.7) are charged under the global symmery $\mathrm{U}(1)_{F N}$ with the charge assignment presented in the table 1 . Note that since the scalar triplets $\rho$ and $\chi$ are in the same representation, the combination $\rho^{\dagger} \chi$ is gauge invariant. Also according to eq. (2.8) and the table 1 , the gauge invariant combination $\rho^{\dagger} \chi$ carries a non-zero FN-charge and has a non-zero VEV. Therefore the $\rho^{\dagger} \chi$-combination can play the role of the flavon in the Froggatt-Nielsen mechanism, as was demonstrated in [25, 26]. The Froggatt-Nielsen mechanism can thus be implemented without introducing new scalar degrees of freedom into the model.

The scalar potential is greatly simplified due to inclusion of global $\mathrm{U}(1)_{F N}$-symmetry. The most general $\mathrm{U}(1)_{F N}$-symmetric scalar potential is,

$$
\begin{aligned}
V_{\mathrm{FN}}= & \mu_{1}^{2} \eta^{\dagger} \eta+\mu_{2}^{2} \rho^{\dagger} \rho+\mu_{3}^{2} \chi^{\dagger} \chi+\lambda_{1}\left(\eta^{\dagger} \eta\right)^{2}+\lambda_{2}\left(\rho^{\dagger} \rho\right)^{2}+\lambda_{3}\left(\chi^{\dagger} \chi\right)^{2} \\
& +\lambda_{12}\left(\eta^{\dagger} \eta\right)\left(\rho^{\dagger} \rho\right)+\lambda_{13}\left(\eta^{\dagger} \eta\right)\left(\chi^{\dagger} \chi\right)+\lambda_{23}\left(\rho^{\dagger} \rho\right)\left(\chi^{\dagger} \chi\right) \\
& +\widetilde{\lambda}_{12}\left(\eta^{\dagger} \rho\right)\left(\rho^{\dagger} \eta\right)+\widetilde{\lambda}_{13}\left(\eta^{\dagger} \chi\right)\left(\chi^{\dagger} \eta\right)+\widetilde{\lambda}_{23}\left(\rho^{\dagger} \chi\right)\left(\chi^{\dagger} \rho\right) \\
& +\sqrt{2} f\left(\epsilon_{i j k} \eta^{i} \rho^{j} \chi^{k}+\text { h.c. }\right) .
\end{aligned}
$$

However the global $\mathrm{U}(1)_{F N}$-symmetry is spontaneously broken by the scalar field VEVs. This leaves one Golstone boson to the physical spectrum. In order to give it a mass we add the following soft FN-breaking term to the potential:

$$
V_{\text {soft }}=b\left(\rho^{\dagger} \chi\right)+\text { h.c. }
$$

All the complex phases in the scalar potential can be absorbed into the fields and therefore all the parameters in the scalar potential are real. The real and imaginary parts of the scalars will therefore not mix. We choose the parameter $f$ to be comparable to the $\mathrm{SU}(3)_{L} \times \mathrm{U}(1)_{X}$-breaking scale. The soft-breaking term is also chosen to be large, $b \sim-\left(v_{\text {heavy }}\right)^{2}$, in order to decouple the pseudo-Goldstone boson in the low energies. 
The scalar sector has five CP-even, five CP-odd and four charged scalars. One CPeven, three CP-odd and two charged scalars are massless would-be-Goldstone bosons that are absorbed by the gauge bosons of the model, namely the $Z, W^{ \pm}$of the SM, new heavy charged gauge boson $V^{ \pm}$, new heavy neutral gauge boson $Z^{\prime}$ and a non-hermitian heavy neutral gauge boson $X^{0}$. Thus there are four CP-even, two CP-odd and two charged scalars left in the physical spectrum. All the physical scalars, except the $125 \mathrm{GeV}$ scalar, have their masses around the $\mathrm{SU}(3)_{L} \times \mathrm{U}(1)_{X}$-breaking scale and they are very heavy. The details of the scalar mass matrices are provided in the appendix A.

\subsection{Gauge sector}

As previously mentioned the gauge sector of 331-model is enlarged compared to the SM. The 331-models will contain five additional gauge bosons compared to the SM. The covariant derivative for $\mathrm{SU}(3)_{L}$ triplet is:

$$
D_{\mu}=\partial_{\mu}-i g_{3} \sum_{a=1}^{8} T_{a} W_{a \mu}-i g_{x} X B_{\mu}
$$

where $g_{3}$ and $g_{x}$ are the $\mathrm{SU}(3)_{L}$ and $\mathrm{U}(1)_{X}$ gauge couplings respectively. The $T_{a}=\lambda_{a} / 2$ are the $\mathrm{SU}(3)_{L}$ generators, where $\lambda_{a}$ are the Gell-Mann matrices. The $\mathrm{SU}(3)_{L}$ gauge bosons are,

$$
\sum_{a=1}^{8} T_{a} W_{a \mu}=\frac{1}{\sqrt{2}}\left(\begin{array}{ccc}
\frac{1}{\sqrt{2}} W_{3 \mu}+\frac{1}{\sqrt{6}} W_{8 \mu} & W_{\mu}^{\prime+} & X_{\mu}^{\prime 0} \\
W_{\mu}^{\prime-} & -\frac{1}{\sqrt{2}} W_{3 \mu}+\frac{1}{\sqrt{6}} W_{8 \mu} & V_{\mu}^{\prime-} \\
X_{\mu}^{\prime 0 *} & V_{\mu}^{\prime+} & -\frac{2}{\sqrt{6}} W_{8 \mu}
\end{array}\right)
$$

where we have denoted,

$$
\begin{aligned}
W_{\mu}^{\prime \pm} & =\frac{1}{\sqrt{2}}\left(W_{1 \mu} \mp i W_{2 \mu}\right), \\
V_{\mu}^{\prime \mp} & =\frac{1}{\sqrt{2}}\left(W_{6 \mu} \mp i W_{7 \mu}\right), \\
X_{\mu}^{\prime 0} & =\frac{1}{\sqrt{2}}\left(W_{4 \mu}-i W_{5 \mu}\right) .
\end{aligned}
$$

The fields $W_{3 \mu}, W_{8 \mu}, B_{\mu}$ and $W_{4 \mu}$ will form neutral mass eigenstates: photon, $Z$-boson and new heavy gauge bosons $Z^{\prime}$ and $\widetilde{W}_{4 \mu}$. The field $W_{5 \mu}$ does not mix with the other neutral gauge bosons and is a mass eigenstate, with same mass as $\widetilde{W}_{4 \mu}$. These fields are identified as a physical neutral non-hermitian gauge boson $X_{\mu}^{0} \equiv \frac{1}{\sqrt{2}}\left(\widetilde{W}_{4 \mu}-i W_{5 \mu}\right)$. Details of the neutral gauge boson masses are given in the appendix B. The off-diagonal gauge bosons $W_{\mu}^{\prime \pm}$ and $V_{\mu}^{\prime \pm}$ will form the SM gauge bosons $W_{\mu}^{ \pm}$and the heavy new gauge bosons $V_{\mu}^{ \pm}$.

\subsubsection{Charged gauge bosons}

The mass term for the charged gauge bosons is given by,

$$
\mathcal{L} \supset Y^{T} M_{\text {charged }}^{2} Y,
$$


where $Y^{T}=\left(W_{\mu}^{\prime+}, V_{\mu}^{\prime+}\right)$ and,

$$
M_{\text {charged }}^{2}=\left(\begin{array}{cc}
\frac{g_{3}^{2}}{4}\left(v^{\prime 2}+v_{1}^{2}\right) & \frac{g_{3}^{2} v_{1} v_{2}}{4} \\
\frac{g_{3}^{2} v_{1} v_{2}}{4} & \frac{g_{3}^{2}}{4}\left(v^{\prime 2}+v_{2}^{2}+u^{2}\right)
\end{array}\right),
$$

is the charged gauge boson mass matrix. The eigenvalues of the matrix are,

$$
\begin{aligned}
& m_{W^{ \pm}}^{2}=\frac{g_{3}^{2}}{4}\left(v^{\prime 2}+\frac{v_{1}^{2} u^{2}}{v_{2}^{2}+u^{2}}\right)+\mathcal{O}\left(\frac{v_{\text {light }}^{2}}{v_{\text {heavy }}^{2}}\right), \\
& m_{V^{ \pm}}^{2}=\frac{g_{3}^{2}}{4}\left(v_{2}^{2}+u^{2}\right)+\mathcal{O}\left(\frac{v_{\text {light }}^{2}}{v_{\text {heavy }}^{2}}\right),
\end{aligned}
$$

where $v_{\text {heavy }}=v_{2}, u$ and $v_{\text {light }}=v_{1}, v^{\prime}$. According to eq. (2.13) the SM Higgs VEV is related to the triplet VEVs through the relation

$$
v^{\prime 2}+\frac{v_{1}^{2} u^{2}}{v_{2}^{2}+u^{2}}+\mathcal{O}\left(\frac{v_{\text {light }}^{2}}{v_{\text {heavy }}^{2}}\right)=\left(v_{s m}\right)^{2},
$$

where $v_{s m}=246 \mathrm{GeV}$.

The mass eigenstates are defined as

$$
\left\{\begin{array}{l}
W_{\mu}^{+}=\cos \theta W_{\mu}^{\prime+}+\sin \theta V_{\mu}^{\prime+} \\
V_{\mu}^{+}=-\sin \theta W_{\mu}^{\prime+}+\cos \theta V_{\mu}^{\prime+}
\end{array},\right.
$$

where the mixing angle $\theta$ is defined as:

$$
\tan 2 \theta=-\frac{2 v_{1} v_{2}}{v_{2}^{2}+u^{2}-v_{1}^{2}}
$$

The mixing angle between $W_{\mu}^{ \pm}$and $V_{\mu}^{ \pm}$is tiny, $\theta \sim \frac{v_{\text {light }}}{v_{\text {heavy }}}$, due to large difference between the $\mathrm{SU}(3)_{L} \times \mathrm{U}(1)_{X}$ and $\mathrm{SU}(2)_{L} \times \mathrm{U}(1)_{Y}$-breaking VEVs. The SM gauge boson $W_{\mu}^{ \pm}$is almost totally $W_{\mu}^{\prime \pm}$ and $V_{\mu}^{ \pm}$is mostly $V_{\mu}^{\prime \pm}$. The experimental bound for the mixing is $|\theta| \lesssim \mathcal{O}\left(10^{-2}\right)[1]$, and has been taken into account in our numerical analysis (section 8).

\section{The Yukawa sector and the Froggatt-Nielsen mechanism in the 331- framework}

Next we study the Yukawa sector of the model. We are employing the Froggatt-Nielsen mechanism to generate the hierarchical structure of the fermion Yukawa couplings. The original Froggatt-Nielsen model extends the Standard Model with a flavour symmetry (FN symmetry), whose symmetry group in the simplest case is global or local U(1) or a discrete $Z_{N}$ symmetry. The FN-framework introduces a new complex scalar field, the flavon, which is a singlet under standard model gauge group $\mathrm{SU}(3)_{C} \times \mathrm{SU}(2)_{L} \times \mathrm{U}(1)_{Y}$. The SM fermions, the SM Higgs and the flavon are charged under the FN symmery. The key property of the FN-symmetry is to forbid the SM Yukawa couplings, save perhaps the top quark. The SM Yukawa couplings are generated as effective couplings instead. 


\begin{tabular}{|c|c|c|c|}
\hline Particle & $\left(\psi_{L, i}^{f}\right)^{c}$ & $f_{R, i}$ & $S$ \\
\hline FN charge & $q\left(\bar{\psi}_{L, i}^{f}\right)$ & $q\left(f_{R, j}\right)$ & $q_{S}$ \\
\hline
\end{tabular}

Table 2. The FN charges of fermions and the scalar fields.

The FN mechanism can be economically incorporated into a 331-model. The scalar sector we have introduced in section 2.2 contains the neccesary incredients to house FroggattNielsen mechanism, as was demonstrated in $[25,26]$. The addition of complex scalar field to act as a flavon is thus unneccesary. Here a gauge singlet combination, $\rho^{\dagger} \chi$, will act as the flavon instead of single complex scalar field. The effective flavon, $\rho^{\dagger} \chi$, obtains a nonzero vacuum expectation value, $\left\langle\rho^{\dagger} \chi\right\rangle=\left(v_{2} u\right) / 2$, as can be seen from the eq. (2.8).

The effective operator that generates the Yukawa couplings of the charged fermions is: ${ }^{3}$

$$
\mathcal{L} \supset\left(c_{s}^{f}\right)_{i j}\left(\frac{\rho^{\dagger} \chi}{\Lambda^{2}}\right)^{\left(n_{f}^{s}\right)_{i j}} \bar{\psi}_{L, i}^{f} S f_{R, j}+\text { h.c. }
$$

where $\left(c_{s}^{f}\right)_{i j}$ is a dimensionless order-one number, $\Lambda$ is the scale of the new physics, $S$ denotes any of the three scalar triplets $\eta, \rho$ or $\chi$. The $\bar{\psi}_{L, i}^{f}$ and $f_{R, j}$ represent here the fermion triplets, antitriplets and singlets that were introduced in section 2.1. The power $\left(n_{f}^{s}\right)_{i j}$ is positive integer number ${ }^{4}$ and determined by the FN charge conservation (see table 2):

$$
\left(n_{f}^{s}\right)_{i j}=\left[q\left(\bar{\psi}_{L, i}^{f}\right)+q\left(f_{R, j}\right)+q(S)\right] .
$$

The usual 331-model Yukawa terms are generated as effective couplings when the scalar triplets $\rho$ and $\chi$ acquire VEVs:

$$
\begin{aligned}
\left(c_{s}^{f}\right)_{i j} & \left(\frac{(\rho+\langle\rho\rangle)^{\dagger}(\chi+\langle\chi\rangle)}{\Lambda^{2}}\right)^{\left(n_{f}^{s}\right)_{i j}} \bar{\psi}_{L, i}^{f}(S+\langle S\rangle) f_{R, j}+\text { h.c. } \\
= & \left(y_{s}^{f}\right)_{i j} \bar{\psi}_{L, i}^{f}(S+\langle S\rangle) f_{R, j}+\left(n_{f}^{s}\right)_{i j}\left(y_{s}^{f}\right)_{i j}\left[\frac{\rho^{\prime 0 *}}{v_{2}}+\frac{\chi^{\prime 0}}{u}+\frac{v_{1} \chi^{0}}{v_{2} u}\right] \sqrt{2} \bar{\psi}_{L, i}^{f}\langle S\rangle f_{R, j} \\
& \quad+\text { h.c. }+\cdots
\end{aligned}
$$

where only the renormalizable contributions are kept. The first term in eq. (3.3) gives the usual Yukawa terms of the model, as in the original FN model. The Yukawa coupling is now defined as:

$$
\left(y_{s}^{f}\right)_{i j}=\left(c_{s}^{f}\right)_{i j}\left(\frac{v_{2} u}{2 \Lambda^{2}}\right)^{\left(n_{f}^{s}\right)_{i j}} \equiv\left(c_{s}^{f}\right)_{i j} \epsilon^{\left(n_{f}^{s}\right)_{i j}} .
$$

Hierarchical Yukawa couplings are produced by assuming that $\epsilon=\left(v_{2} u\right) /\left(2 \Lambda^{2}\right)<1$. The FN-charges of the SM fermions determine the power $\left(n_{s}^{f}\right)_{i j}$ and therefore the amount of suppression each Yukawa coupling obtains. One can obtain the observed fermion mass hierarchy by assigning larger FN charges to the lighter fermions compared to the heavier

\footnotetext{
${ }^{3}$ The effective operator that generates the neutrino Yukawa couplings is presented later in eq. (5.1).

${ }^{4}$ If $\left(n_{f}^{s}\right)_{i j}$ were negative, we would have to include operator $\left(c_{s}^{f}\right)_{i j}\left(\frac{\chi^{\dagger} \rho}{\Lambda^{2}}\right)^{-\left(n_{f}^{s}\right)_{i j}} \bar{\psi}_{L, i}^{f} S f_{R, j}+$ h.c.
} 
ones. This is in contrast to the Standard Model where the hierarchy is obtained only by fine-tuning the couplings themselves.

The second term in eq. (3.3) is not proportional to the Yukawa matrix and is therefore flavour violating. This flavour violating part is suppressed by the scale of the $\mathrm{SU}(3)_{L} \times$ $\mathrm{U}(1)_{X}$-breaking. We assume that the $\mathrm{SU}(3)_{L} \times \mathrm{U}(1)_{X}$-breaking scale $v_{\text {scale }}$ is $\gtrsim 7 \mathrm{TeV}$. This is enough to suppress the quark FCNCs as shown in [26]. This also ensures that the $Z^{\prime}$ mass is $\gtrsim 3 \mathrm{TeV}$, satisfying bounds in [27]. We will safely ignore the flavour violating contributions as they are heavily suppressed.

\section{Charged lepton Yukawa couplings and masses}

Charged lepton Yukawa couplings are:

$$
\mathcal{L}_{\text {lepton }}=y_{i j}^{e} \bar{L}_{L, i} \eta e_{R, j}+l_{i j} y_{i j}^{e} \bar{e}_{L, i} e_{R, j}\left[\frac{v^{\prime}}{v_{2}} \rho^{\prime 0 *}+\frac{v^{\prime}}{u} \chi^{\prime 0}+\frac{v^{\prime} v_{1}}{v_{2} u} \chi^{0}\right]+\text { h.c. },
$$

where $i, j=1,2,3$. The first term is the traditional 331 Yukawa term for the charged leptons whereas the second term is the additional Yukawa interaction term due to the FNmechanism. As stated earlier, this additional term is suppressed by the $v_{\text {heavy }}$ and we will ignore it in the following. The charged lepton Yukawa matrix is given as follows:

$$
y_{i j}^{e}=c_{i j}^{e} \epsilon^{q\left(\bar{L}_{L, i}\right)+q\left(e_{R, j}\right)+q(\eta)} \equiv c_{i j}^{e} \epsilon^{l_{i j}} .
$$

We will specify the FN-charges we use later in section 8 , when we study numerical examples.

The charged leptons acquire masses as the scalar triplet $\eta$ obtains a VEV:

$$
\mathcal{L} \supset y_{i j}^{e} \bar{L}_{L, i}\langle\eta\rangle e_{R, j}+\text { h.c. }=m_{i j}^{e} \bar{e}_{L, i} e_{R, j}+\text { h.c. },
$$

where the charged lepton mass matrix is,

$$
m_{i j}^{e}=y_{i j}^{e} \frac{v^{\prime}}{\sqrt{2}} .
$$

The charged lepton mass matrix is diagonalized as:

$$
U_{L}^{e} m^{e} U_{R}^{e \dagger}=m_{\text {diag }}^{e} .
$$

The charged lepton mass matrix proportional to the Yukawa matrix will be diagonalized simultaneously with the Yukawa coupling. Therefore there will be no flavour changing couplings in the standard Yukawa couplings. The only flavour violation to the charged leptons is coming from the Froggatt-Nielsen mechanism which is however suppressed.

\section{$5 \quad$ Neutrino mass matrix}

The neutrino Yukawa couplings originate from effective operators of two types. The first type was already presented in eq. (3.1) and the operator of the second kind is:

$$
\mathcal{L} \supset\left(c_{\eta^{*}}^{N}\right)_{i j}\left(\frac{\rho^{\dagger} \chi}{\Lambda^{2}}\right)^{\left(n_{N}^{\eta^{*}}\right)_{i j}} \epsilon_{\alpha \beta \gamma} \bar{L}_{L, i}^{\alpha}\left(L_{L, j}^{c}\right)^{\beta}\left(\eta^{*}\right)^{\gamma}+\text { h.c. }
$$


The operators in eqs. (3.1) and (5.1) produce the following Yukawa couplings for neutrinos:

$$
\begin{aligned}
\mathcal{L}_{\text {neutrino }}= & e_{i j} \epsilon_{\alpha \beta \gamma} \bar{L}_{L, i}^{\alpha}\left(L_{L, j}^{c}\right)^{\beta}\left(\eta^{*}\right)^{\gamma}+y_{i j}^{N} \bar{L}_{L, i} \rho N_{R, j}+y_{i j}^{\prime N} \bar{L}_{L, i} \chi N_{R, j} \\
& +\left\{\left(n_{N}^{\eta^{*}}\right)_{i j} e_{i j} \epsilon_{\alpha \beta \gamma} \bar{L}_{L, i}^{\alpha}\left(L_{L, j}^{c}\right)^{\beta}\left\langle\eta^{*}\right\rangle^{\gamma}+n_{i j} y_{i j}^{N} \bar{L}_{L, i}^{\prime}\langle\rho\rangle N_{R, j}^{\prime}\right. \\
& \left.+n_{i j}^{\prime} y_{i j}^{\prime N} \bar{L}_{L, i}^{\prime}\langle\chi\rangle N_{R, j}^{\prime}\right\} \sqrt{2}\left[\frac{\rho^{\prime 0 *}}{v_{2}}+\frac{\chi^{\prime 0}}{u}+\frac{v_{1}}{v_{2} u} \chi^{0}\right]+\text { h.c. },
\end{aligned}
$$

where $i, j=1,2,3$ and the Yukawa couplings are,

$$
\begin{aligned}
& y_{i j}^{N}=c_{i j}^{N} \epsilon^{q\left(\bar{L}_{L, i}\right)+q\left(N_{R, j}\right)+q(\rho)}=c_{i j}^{N} \epsilon^{n_{i j}}, \\
& y^{\prime N}=c_{i j}^{\prime N} \epsilon^{q\left(\bar{L}_{L, i}\right)+q\left(N_{R, j}\right)+q(\chi)}=c_{i j}^{\prime N} \epsilon^{n_{i j}^{\prime}}, \\
& e_{i j}=\left(c_{\eta^{*}}^{N}\right)_{i j} \epsilon^{q\left(L_{L, i}^{c}\right)+q\left(L_{L, j}^{c}\right)+q\left(\eta^{*}\right)}=\left(c_{\eta^{*}}^{N}\right)_{i j} \epsilon^{\left(n_{N}^{\eta^{*}}\right)_{i j}} .
\end{aligned}
$$

The Yukawa coupling $e_{i j}$ is antisymmetric: $e_{i j}=-e_{j i}$, due to presence of the antisymmetric tensor $\epsilon_{\alpha \beta \gamma}$ in the eq. (5.2).

The first line in (5.2) contains the standard Yukawa interactions for the neutrinos and the two last lines contain the additional Yukawa interactions originating from the Froggatt-Nielsen mechanism, which we will ignore due to them being suppressed.

The neutrino masses will be generated by the Yukawa terms in the first line of eq. (5.2) as the scalars obtain VEVs. The right-handed neutrino singlet $N_{R, i}$ will also have a Majorana mass term which is generated by the following operator,

$$
\mathcal{L} \supset M_{0} c_{i j}^{M}\left(\frac{\rho^{\dagger} \chi}{\Lambda^{2}}\right)^{q\left(N_{R, i}\right)+q\left(N_{R, j}\right)} \overline{\left(N_{R, j}\right)^{c}} N_{R, j}+\text { h.c. },
$$

where the mass scale $M_{0}$ is in principle a free parameter. We choose the mass scale to be same as the FN-messengers, in order not to introduce new mass scales into the model. The Majorana mass term becomes,

$$
\mathcal{L}_{\text {Majorana }}=\frac{1}{2} M_{i j} \overline{\left(N_{R, j}\right)^{c}} N_{R, j}+\text { h.c. }
$$

where the Majorana mass matrix is,

$$
M_{i j}=\Lambda c_{i j}^{M} \epsilon^{q\left(N_{R, i}\right)+q\left(N_{R, j}\right)} .
$$

The messenger scale is related to the $\mathrm{SU}(3)_{L} \times \mathrm{U}(1)_{X}$-breaking VEVs by,

$$
\Lambda=\sqrt{\frac{u v_{2}}{2 \epsilon}} .
$$

The full contribution to the neutrino masses is finally given by the following terms:

$$
\begin{aligned}
\mathcal{L}_{\text {neutrino mass }}= & e_{i j} \epsilon_{\alpha \beta \gamma} \bar{L}_{L, i}^{\alpha}\left(L_{L, j}^{c}\right)^{\beta}\left(\left\langle\eta^{*}\right\rangle\right)^{\gamma}+y_{i j}^{N} \bar{L}_{L, i}\langle\rho\rangle N_{R, j} \\
& +y^{\prime N}{ }_{i j} \bar{L}_{L, i}\langle\chi\rangle N_{R, j}+\frac{1}{2} M_{i j} \overline{\left(N_{R, j}\right)^{c}} N_{R, j}+\text { h.c. }
\end{aligned}
$$


The neutrino masses can be written in a $9 \times 9$ matrix form as:

$$
\begin{aligned}
\mathcal{L}_{\text {neutrino mass }} & =\frac{1}{2}\left(\overline{\left(\nu_{L}\right)^{c}} \overline{\left(\nu^{\prime}{ }_{L}\right)^{c}} \overline{N_{R}}\right)\left(\begin{array}{ccc}
0 & 2\left(m^{D}\right)^{\dagger} & \left(m^{N}\right)^{*} \\
2\left(m^{D}\right)^{*} & 0 & \left(m^{\prime N}\right)^{*} \\
\left(m^{N}\right)^{\dagger} & \left(m^{\prime N}\right)^{\dagger} & M^{*}
\end{array}\right)\left(\begin{array}{c}
\nu_{L} \\
\nu_{L}^{\prime} \\
\left(N_{R}\right)^{c}
\end{array}\right)+\text { h.c. } \\
& \equiv \frac{1}{2} \overline{\left(N^{\prime}\right)^{c}} M_{\nu} N^{\prime}+\text { h.c. }
\end{aligned}
$$

where the $3 \times 3$ sub-matrices are:

$$
m_{i j}^{N}=\frac{v_{1}}{\sqrt{2}} y_{i j}^{N}, \quad{m^{\prime}}_{i j}^{N}=\frac{v_{2}}{\sqrt{2}} y_{i j}^{N}+\frac{u}{\sqrt{2}} y_{i j}^{\prime N} \quad \text { and } \quad m_{i j}^{D}=\frac{v^{\prime}}{\sqrt{2}} e_{i j} \quad i=1,2,3 .
$$

The mass matrix with same structure as in eq. (5.9) has been studied in the literature in [28].

We next determine the pattern of FN-charges for the leptons using the experimental values of the PMNS matrix as guidance. Once the FN-charges are known, the exact hierarchy of the neutrino mass matrix becomes clear, and we can proceed with the block diagonalization of the neutrino mass matrix.

\section{$6 \quad$ Neutrino masses and eigenstates}

The Froggatt-Nielsen charges determine the hierarchy of the fermion mass matrices. The fermion FN-charges should be chosen so that the order of magnitude of the fermion masses becomes right, thus the mass hierarchy is explained without fine-tuning. The FN charges also determine the structure of the matrices that diagonalize the fermion mass matrix. This is important as the left-handed fermion diagonalization matrices enter the two physical observables: the CKM-matrix and the PMNS-matrix. Proper choice of the left-handed fermion FN-charges can ensure that also the hierarchy of the CKM- and PMNS-matrices are produced correctly, and no fine-tuning is required. The quark sector of our model is identical to the one in $[25,26]$, where it was studied in great detail. We will therefore not consider it here. We instead concentrate on lepton sector which differs from the model presented in $[25,26]$ only by the additional neutrino singlets $N_{R, i}$.

The determining factor in our choice of leptonic FN-charges is the form of the PMNS matrix. The current experimental values of the PMNS-matrix elements by the NuFit collaboration are:

$$
\left|U_{\mathrm{PMNS}}\right|=\left(\begin{array}{ccc}
0.797-0.840 & 0.518-0.585 & 0.143-0.156 \\
0.233-0.495 & 0.448-0.679 & 0.639-0.783 \\
0.287-0.532 & 0.486-0.706 & 0.604-0.754
\end{array}\right),
$$

where the value of each entry is given at $3 \sigma$ confidence level $[35,36]$.

The PMNS-matrix elements are $\mathcal{O}(1)$ numbers in contrast to CKM-matrix where distinct hierarchy is present. The PMNS-matrix is given schematically by the left-handed charged lepton diagonalization matrix $U_{L}^{e}$ and the neutrino diagonalization matrix $U_{\nu}$ as: ${ }^{5}$

$$
U_{\mathrm{PMNS}} \sim U_{L}^{e} U_{\nu}
$$

\footnotetext{
${ }^{5}$ The exact form of the PMNS matrix is given later in eq. (7.4).
} 
The observed PMNS-hierarchy is naturally obtained, if the left-handed charged lepton rotation matrix $U_{L}^{e}$ and neutrino diagonalization matrix $U_{\nu}$, also have this anarchical texture. This is the method we adopt here. The hierarchy of $U_{L}^{e}$ and $U_{\nu}$ depend on the FN-charges of the left-handed leptons. The anarchical hierarchy is achieved when all the lepton families are treated equally under the FN-symmetry. We will therefore choose from now on all the lepton triplets to have equal FN-charges:

$$
q\left(L_{L, 1}^{c}\right)=q\left(L_{L, 2}^{c}\right)=q\left(L_{L, 3}^{c}\right) \equiv L .
$$

The FN-charges of the right-handed neutrino singlets do not affect the hierarchy of the light-neutrinos. We will choose the FN-charge of the right-handed neutrinos to be zero for simplicity:

$$
q\left(N_{R, 1}\right)=q\left(N_{R, 2}\right)=q\left(N_{R, 3}\right)=0 .
$$

We can now see the order of magnitude in the neutrino mass matrix elements and proceed with the block diagonalization of the neutrino mass matrix.

\subsection{Neutrino mass matrices}

The neutrino mass matrix $M_{\nu}$ in eq. (5.9) will have nine eigenvalues corresponding to nine Majorana neutrinos. The neutrino mass matrix $M_{\nu}$ can be written in the following notation:

$$
M_{\nu}=\left(\begin{array}{cc}
0 & \mathbb{M}_{\mathbb{D}}^{\mathbb{T}} \\
\mathbb{M}_{\mathbb{D}} & \mathbb{M}_{\mathbb{R}}
\end{array}\right)
$$

where

$$
\mathbb{M}_{\mathbb{D}}^{\mathbb{T}}=\left(\begin{array}{ll}
2\left(m^{D}\right)^{\dagger} & \left(m^{N}\right)^{*}
\end{array}\right) \quad \text { and } \quad \mathbb{M}_{\mathbb{R}}=\left(\begin{array}{cc}
0 & \left(m^{\prime N}\right)^{*} \\
\left(m^{\prime N}\right)^{\dagger} & M^{*}
\end{array}\right) .
$$

The order of magnitude of the sub-matrices are given by

$$
m_{i j}^{D} \sim v_{\text {light }} \epsilon^{2 L+1}, \quad m_{i j}^{N} \sim v_{\text {light }} \epsilon^{L+1}, \quad m_{i j}^{\prime N} \sim v_{\text {heavy }} \epsilon^{L} \quad \text { and } \quad M_{i j} \sim v_{\text {heavy }},
$$

where $v_{\text {light }}=v^{\prime}, v_{1}$ and $v_{\text {heavy }}=u, v_{2}$. Note that sub-matrices in eq. (6.7) do not have an internal hierarchy, but distinct hierarchy is present between sub-matrices $\mathbb{M}_{\mathbb{D}}$ and $\mathbb{M}_{\mathbb{R}}$. The entries in the sub-matrix $\mathbb{M}_{\mathbb{R}}$ are proportional to the $\mathrm{SU}(3)_{L} \times \mathrm{U}(1)_{X}$ breaking VEVs, $u$ and $v_{2}$, whereas the entries in the sub-matrix $\mathbb{M}_{\mathbb{D}}$ are proportional to $\mathrm{SU}(2)_{L} \times \mathrm{U}(1)_{Y}$ breaking VEVs $v^{\prime}$ and $v_{1}$. Therefore the eigenvalues of the $\mathbb{M}_{\mathbb{R}}$ are much larger than the entries in the $\mathbb{M}_{\mathbb{D}}$. This hierarchy is reflected in the eigenvalues of the matrix: it has three "light" and six heavier eigenvalues.

The elements in the heavier block $\mathbb{M}_{\mathbb{R}}$ also have different orders of magnitude: matrix $m^{\prime N}$ is heavily suppressed compared to $M$ by $\epsilon^{L}$. The eigenvalues of the heavier block will therefore be in two distinct scales we call "medium" and "heavy". Our neutrino sector is subject to kind of "double-seesaw". The neutrino mass matrix $M_{\nu}$ will have in total three "light" eigenvalues, three "medium" eigenvalues and three "heavy" eigenvalues. 
The neutrino mass matrix $M_{\nu}$ can be block-diagonalized into three blocks, each corresponding to these eigenvalue-types according to:

$$
Z^{T} W^{T} M_{\nu} W Z=\left(\begin{array}{ccc}
m_{\text {light }}^{3 \times 3} & 0_{3 \times 3} & 0_{3 \times 3} \\
0_{3 \times 3} & m_{\text {medium }}^{3 \times 3} & 0_{3 \times 3} \\
0_{3 \times 3} & 0_{3 \times 3} & m_{\text {heavy }}^{3 \times 3}
\end{array}\right),
$$

where unitary matrix $W$ separates the three "light"-neutrinos from the six heavier ones, and unitary matrix $Z$ further block diagonalizes the block of heavier neutrinos into block of "medium"-mass neutrinos and "heavy" neutrinos. The matrices $W$ and $Z$ are to the leading order:

$$
W=\left(\begin{array}{cc}
\left(1-\frac{1}{2} B_{1} B_{1}^{\dagger}\right)_{3 \times 3} & \left(B_{1}\right)_{3 \times 6} \\
-\left(B_{1}^{\dagger}\right)_{6 \times 3} & \left(1-\frac{1}{2} B_{1}^{\dagger} B_{1}\right)_{6 \times 6}
\end{array}\right),
$$

and,

$$
Z=\left(\begin{array}{ccc}
1_{3 \times 3} & 0_{3 \times 3} & 0_{3 \times 3} \\
0_{3 \times 3} & \left(1-\frac{1}{2} C_{1} C_{1}^{\dagger}\right)_{3 \times 3} & \left(C_{1}\right)_{3 \times 3} \\
0_{3 \times 3} & -\left(C_{1}^{\dagger}\right)_{3 \times 3} & \left(1-\frac{1}{2} C_{1}^{\dagger} C_{1}\right)_{3 \times 3}
\end{array}\right)
$$

with,

$$
\begin{aligned}
\left(B_{1}\right)_{3 \times 6} & =\left(\begin{array}{ll}
-2\left(m^{D}\right)^{T}\left(\left(m^{\prime N}\right)^{T}\right)^{-1} M\left(m^{\prime N}\right)^{-1}+\left(m^{N}\right)\left(m^{\prime N}\right)^{-1} & 2\left(m^{D}\right)^{T}\left(\left(m^{\prime N}\right)^{T}\right)^{-1}
\end{array}\right)_{3 \times 6} \\
& =\left(\begin{array}{ll}
\left(B_{1}^{1}\right)_{3 \times 3} & \left(B_{1}^{2}\right)_{3 \times 3}
\end{array}\right),
\end{aligned}
$$

and,

$$
C_{1}=m^{\prime N} M^{-1}
$$

The light, medium and heavy blocks can be written at lowest order as:

$$
\begin{aligned}
m_{\text {light }}= & 2 m^{D \dagger}\left(m^{\prime N \dagger}\right)^{-1} M^{*}\left(m^{\prime N *}\right)^{-1} 2 m^{D *} \\
& -\left[m^{N *}\left(m^{\prime N *}\right)^{-1} 2 m^{D *}+2 m^{D \dagger}\left(m^{\prime N \dagger}\right)^{-1} m^{N \dagger}\right] \\
m_{\text {medium }}= & m^{\prime N *}\left(M^{*}\right)^{-1} m^{\prime N \dagger} \\
m_{\text {heavy }}= & M^{*} .
\end{aligned}
$$

The order of magnitude of light-, medium- and heavy-neutrino masses can now be estimated using eq. (6.13) with eq. (6.7):

$$
m_{\text {light }, i j} \sim \frac{v_{\text {light }}^{2}}{v_{\text {heavy }}} \epsilon^{2 L+2}, \quad m_{\text {medium }, i j} \sim v_{\text {heavy }} \epsilon^{2 L}, \quad \text { and } \quad m_{\text {heavy }, i j} \sim v_{\text {heavy }} .
$$

The light-neutrino masses are proportional to $v_{\text {light }}^{2} / v_{\text {heavy }}$, where $v_{\text {light }}$ is the electroweak scale and $v_{\text {heavy }}$ is the scale of new physics, which is characteristic to the seesaw-mechanism. Additional suppression factor, $\epsilon^{2 L+2}$, is however present, due to the Froggatt-Nielsen mechanism. The masses of the "medium"-neutrinos are heavily suppressed compared to 
$\mathrm{SU}(3)_{L} \times \mathrm{U}(1)_{X}$-breaking scale, making them typically lighter than $m_{Z} / 2$. They are therefore subject to the LEP bound [1]-[7] on the number of light neutrinos. However, suppression on their couplings to $Z$ boson make their contribution to the invisible decay with of $Z$ boson tiny, as will be demonstrated later for our benchmark points. The heavy neutrinos have their masses around the $\mathrm{SU}(3)_{L} \times \mathrm{U}(1)_{X}$-breaking scale and will therefore decouple.

\subsection{Neutrino eigenstates}

The neutrino mass eigenstates are obtained once the light, medium and heavy neutrino blocks are diagonalized. The neutrino mass matrix $M_{\nu}$ in eq. (5.9) is fully diagonalized according to:

$$
M_{\nu}^{\text {diag }}=\left(U^{T} Z^{T} W^{T}\right) M_{\nu}(W Z U),
$$

with unitary matrix $U$ is given by,

$$
U=\left(\begin{array}{ccc}
U_{\nu}^{3 \times 3} & 0_{3 \times 3} & 0_{3 \times 3} \\
0_{3 \times 3} & U_{n}^{3 \times 3} & 0_{3 \times 3} \\
0_{3 \times 3} & 0_{3 \times 3} & U_{N}^{3 \times 3}
\end{array}\right) .
$$

The unitary matrices $U_{\nu}, U_{n}$ and $U_{N}$ diagonalize light, medium and heavy neutrino blocks respectively. The $U_{\nu}, U_{n}$ and $U_{N}$ are anarchical in nature,

$$
U_{\nu}, U_{n}, U_{N} \sim\left(\begin{array}{lll}
1 & 1 & 1 \\
1 & 1 & 1 \\
1 & 1 & 1
\end{array}\right),
$$

as the blocks $m_{\text {light }}, m_{\text {medium }}$ and $m_{\text {heavy }}$ have no internal hierarchy.

According the eq. (6.15), the mass eigenstate neutrinos are:

$$
\nu_{\text {mass }} \equiv\left(\begin{array}{c}
\nu_{\text {light }, L}^{3 \times 1} \\
\nu_{\text {medium }, L}^{3 \times 1} \\
\nu_{\text {heavy }, L}^{3 \times 1}
\end{array}\right)=U^{\dagger} Z^{\dagger} W^{\dagger} N^{\prime} .
$$

The mixing between the neutrinos can be estimated with the use of FN-textures of the neutrino Yukawa couplings as:

$$
\left\{\begin{array}{l}
\nu_{L}=\mathcal{O}(1) \cdot \nu_{\text {light }, L}+\mathcal{O}\left[\frac{v_{\text {light }}}{v_{\text {heavy }}} \epsilon^{1}\right] \cdot \nu_{\text {medium }, L}+\mathcal{O}\left[\frac{v_{\text {light }}}{v_{\text {heavy }}} \epsilon^{L+1}\right] \cdot \nu_{\text {heavy }, L} \\
\nu_{L}^{\prime}=\mathcal{O}\left[\frac{v_{\text {light }}}{v_{\text {heavy }}} \epsilon^{1}\right] \cdot \nu_{\text {light }, L}+\mathcal{O}(1) \cdot \nu_{\text {medium }, L}+\mathcal{O}\left[\epsilon^{L}\right] \cdot \nu_{\text {heavy }, L} \\
\left(N_{R}\right)^{c}=\mathcal{O}\left[\frac{v_{\text {light }}}{v_{\text {heavy }}} \epsilon^{L+1}\right] \cdot \nu_{\text {light }, L}+\mathcal{O}\left[\epsilon^{L}\right] \cdot \nu_{\text {medium }, L}+\mathcal{O}(1) \cdot \nu_{\text {heavy }, L}
\end{array}\right.
$$

\section{Neutrino coupling to charged gauge bosons and PMNS-matrix}

Our model includes additional charged gauge boson $V_{\mu}^{ \pm}$, that mixes with the $W_{\mu}^{ \pm}$boson as shown in the section 2.3.1. The mixing between the charged gauge bosons is however 
tiny. The neutrino gauge eigenstates couple to the physical charged gauge bosons as:

$$
\begin{aligned}
\mathcal{L}_{g C C}= & \frac{g_{3}}{\sqrt{2}}\left[\bar{\nu}_{L, i} \gamma^{\mu} e_{L, i}^{\prime} \cos \theta+\bar{\nu}_{L, i}^{\prime} \gamma^{\mu} e_{L, i}^{\prime} \sin \theta\right] W_{\mu}^{+} \\
& +\frac{g_{3}}{\sqrt{2}}\left[-\bar{\nu}_{L, i} \gamma^{\mu} e_{L, i}^{\prime} \sin \theta+\bar{\nu}_{L, i}^{\prime} \gamma^{\mu} e_{L, i}^{\prime} \cos \theta\right] V_{\mu}^{+}+\text {h.c. }
\end{aligned}
$$

With the use of eqs. (4.5) and (6.18) the coupling of $W_{\mu}^{ \pm}$to light neutrinos can be writen as,

$$
\mathcal{L}_{g C C} \supset \frac{g_{3}}{\sqrt{2}} \bar{\nu}_{\text {light }, L} U_{\nu}^{\dagger}\left[\left(1-\frac{1}{2} B_{1} B_{1}^{\dagger}\right) \cos \theta-B_{1}^{1} \sin \theta\right] U_{L}^{e \dagger} \gamma^{\mu} e_{L} W_{\mu}^{+}+\text {h.c. },
$$

from which we can identify the PMNS matrix:

$$
U_{\mathrm{PMNS}}=\cos \theta U_{L}^{e} U_{\nu}-U_{L}^{e}\left[\frac{1}{2} \cos \theta B_{1} B_{1}^{\dagger}+\sin \theta B_{1}^{1 \dagger}\right] U_{\nu}
$$

The term proportional to $\cos \theta B_{1} B_{1}^{\dagger}$ induces nonunitarity effects to neutrino oscillations, which is an expected effect due to inclusion of sterile neutrinos in the model. Deviation from the unitarity is suppressed by the factor $\mathcal{O}\left(v_{\text {light }}^{2} / v_{\text {heavy }}^{2}\right)$ and is significantly smaller than the current bounds [37-39]. In any case, nonunitary mixing strength of $\geq 10^{-2}$ is ruled out.

The term proportional to $\sin \theta B_{1}^{\dagger}$ is similarly suppressed by a factor $\mathcal{O}\left(v_{\text {light }}^{2} / v_{\text {heavy }}^{2}\right)$, but since $B^{1}$ is not Hermitian, the anti-Hermitian part of it induces neutrino decay. Since the nonunitarity and unstability effects are both small, we shall ignore them in the remainder of this paper.

The PMNS matrix therefore is:

$$
U_{\mathrm{PMNS}} \approx \cos \theta U_{L}^{e} U_{\nu}
$$

We have chosen the $v_{\text {heavy }} \gtrsim 7 \mathrm{TeV}$, which makes the mixing angle $\theta$ very small and $\cos \theta \approx 0.9 \approx 1$. As stated in eq. (6.17) the light-neutrino diagonalization matrix $U_{\nu}$ is anarchical. Since the lepton triplet FN-charges are identical also the left-handed charged lepton diagonalization matrix $U_{L}^{e}$ is without a hierarchy: ${ }^{6}$

$$
U_{L}^{e} \sim\left(\begin{array}{lll}
1 & 1 & 1 \\
1 & 1 & 1 \\
1 & 1 & 1
\end{array}\right)
$$

The texture for the PMNS is therefore anarchical as well,

$$
U_{\mathrm{PMNS}} \sim\left(\begin{array}{lll}
1 & 1 & 1 \\
1 & 1 & 1 \\
1 & 1 & 1
\end{array}\right),
$$

which is compatible with the experimental measurements presented in eq. (6.1). We note here that this is the extent which Froggatt-Nielsen setting can predict the structure of

\footnotetext{
${ }^{6}$ When charged lepton mass matrix satisfies $m_{i, j}^{e} \leq m_{i+1, j}^{e}$, the left-handed diagonalization matrix satisfies: $\left(U_{L}^{e}\right)_{i j} \sim \epsilon^{\left|q\left(L_{L, i}^{c}\right)-q\left(L_{L, j}^{c}\right)\right|}$.
} 
PMNS-matrix. This is in contrast to many models involving more elaborate flavour symmetries in the neutrino sector [40-42]. As of now, the PMNS matrix is consistent with anarchical mixing. It is up to the numerics to acquire the order-one coefficients that produce the correct lepton masses and the PMNS-matrix within the experimental limits, which is the focus of section 8 .

\section{Constraints and numerical examples}

There are many important experimental constraints that have to be taken into account when considering the neutrino sector of any model. Constraints for active neutrinos are the most well-known and restrictive. Least model-dependent is the direct detection bound of $m\left(\nu_{e}\right)$ from electron energy spectrum of tritium $\beta$ decay [43] and data from supernova SN1987a burst. Also, neutrinoless double beta decay experiments [44], cosmic microwave background and growth of large scale structures in the early universe [45] all constrain the upper limits of flavour neutrino masses, and their sum. Cosmological constraints are stricter by one order of magnitude, but are dependent on the cosmological model. In addition, neutrino oscillation experiments provide neutrino mass squared differences, $\Delta m_{21}^{2}$ and $\left|\Delta m_{3 j}\right|^{2}$ (with $j=1,2$ corresponding to inverted and normal mass orderings, respectively) $[35,36]$. From these, a lower bound for two heavier light neutrinos can be deduced, being approximately $9 \mathrm{meV}$ and $50 \mathrm{meV}$. The lightest neutrino state may be massless. Cosmological constraints are $\sum m_{\nu}<0.12 \mathrm{eV}$.

The existence of medium-mass sterile neutrinos at $\mathrm{eV}$ and $\mathrm{keV}$ scale would distort the electron energy spectrum, and different sterile neutrino mass ranges of this distortion can be detected via unstable nuclei, such as ${ }^{3} \mathrm{H},{ }^{20} \mathrm{~F},{ }^{35} \mathrm{~S},{ }^{63} \mathrm{Ni}$ and ${ }^{187} \mathrm{Re}$. Searches for these distortions, i.e. kink searches have discarded large mixings of electron neutrino to mediummass sterile neutrinos $[48,49]$. We will show the constraints from kink searches to one of our benchmark points in figure 1. The heavy $\mathrm{TeV}$-scale sterile neutrinos in principle can be probed with a next-generation collider experiments, if their mixing with active flavours is large. However, our benchmark points correspond to extremely tiny TeV-scale sterile neutrino component to active neutrinos, rendering them completely inaccessible.

Our model predicts neutrinos in three different mass scales: the three sub-eV neutrinos, three heavy, mostly right-handed neutrinos, and three neutrinos with masses between the sub-eV and $\mathrm{SU}(3)_{L} \times \mathrm{U}(1)_{X}$ scales. The masses of the medium-scale neutrinos is determined by the $\mathrm{SU}(3)_{L} \times \mathrm{U}(1)_{X}$-breaking scale and in the case of $v_{\text {heavy }} \sim 50 \mathrm{TeV}$ the medium-scale neutrino masses are around keV scale. The sub-eV neutrinos are constrained by their mass squared differences and mixings. The keV neutrinos that our model predicts are constrained by the LEP bound on the number of light neutrinos, "light" here meaning neutrinos with their masses smaller than $m_{Z} / 2$ [1-7]. The coupling of medium mass neutrinos to $Z$-bosons is heavily suppressed by the ratio between $\mathrm{SU}(2)_{L} \times \mathrm{U}(1)_{Y^{-}}$and $\mathrm{SU}(3)_{L} \times \mathrm{U}(1)_{X}$-breaking scales and they will pass the LEP limits on the number of light neutrinos. This becomes evident in our benchmark points.

Seesaw mechanisms have been successfully applied to the 331 models also previously e.g. $[50,51]$. Although it is not possible to distinguish the models experimentally from 
each other solely by the light neutrino sector, the particle spectra of the models may differ otherwise, for example, [50, 51] contain exotic charged leptons not present in the FN331 model. If the non-Standard Model particles of the model turn out to be within reach of experiments, in the case of the FN331 model, the masses and couplings are related to each other in a calculable way and thus the measurement can hint towards the model.

The tree-level CLFV decays of the charged leptons are heavily suppressed as we have stated in section 4 . The neutrinos will still mediate charged lepton decays at loop-level. These are also heavily suppressed due to small neutrino Yukawa-couplings presented in eq. (5.3) and due to small mixing between neutrinos presented in eq. (6.19). The CLFV decays of charged leptons do not pose constraints to our model, in constrast to some other 331-models such as [52] and [53], which contain additional sources for charged lepton flavour violation such as bileptons. Also in 331-models with inverse seesaw mechanism the CLFV decays of charged leptons can be significant $[54,55]$, in contrast to our model.

The sterile neutrinos of our model come in two distinct mass ranges "medium" and "heavy". In our benchmark points the medium sterile neutrinos are lighter than the charged leptons and cannot decay into them. The heavy sterile neutrinos on the other hand have their masses at 10-100 TeV scale and are not produced in collider experiments and therefore do not pose any bounds on our model. This is in contrast to the model in [55].

Our model possesses the extended particle content of the 331-model. The additional gauge bosons and scalars of our model could potentially mediate the non-standard neutrino interactions. For example the additional charged gauge boson, $V_{\mu}^{ \pm}$, mediates the CC-NSI given by,

$$
\mathcal{L}_{N S I}^{C C}=-2 \sqrt{2} G_{F} \epsilon_{\alpha \beta}^{l l^{\prime}, L}\left(\bar{\nu}_{\text {light }, L, \alpha} \gamma^{\mu} \nu_{l i g h t, L, \beta}\right)\left(\bar{l} \gamma_{\mu} P_{L} l^{\prime}\right) .
$$

The $V_{\mu}^{ \pm}$mediated contribution to the NSI will be heavily suppressed by its mass:

$$
\epsilon_{\alpha \beta}^{l l^{\prime}, L} \sim \frac{v_{s m}^{2}}{m_{V^{ \pm}}^{2}},
$$

making it negligible as $m_{V^{ \pm}} \gtrsim 7 \mathrm{TeV}$. Indeed, for our numerical benchmarks, the NSI parameters have magnitude $\mathcal{O}\left(10^{-13}\right)$. All the new gauge bosons and scalars of our model have their masses proportional to the $\mathrm{SU}(3)_{L} \times \mathrm{U}(1)_{X}$-breaking scale. The non-standard interactions mediated by charged scalars will therefore also be suppressed due to their heavy masses.

\subsection{The FN-charges for the numerical example}

For the numerical example we take the $\mathrm{SU}(3)_{L} \times \mathrm{U}(1)_{X}$-breaking scale $v_{\text {heavy }}$ to be around 7 to $50 \mathrm{TeV}$, as for this scale the quark sector was studied in $[25,26]$. We choose the values for the leptonic FN-charges so that the $\mathrm{SU}(3)_{L} \times \mathrm{U}(1)_{X}$-breaking scale is fixed.

The FN-charge of the left-handed lepton triplet $L_{L, i}$ is determined by the light-neutrino masses. According to eq. (6.14) all the light-neutrino masses $m_{i}$ will be:

$$
m_{i} \sim \frac{v_{\text {light }}^{2}}{v_{\text {heavy }}} \epsilon^{2 L+2},
$$

where the only free parameter is the FN-charge of the lepton-triplet. 
Experimentally the light-neutrino masses are constrained by [35, 36]:

$$
m_{1}<0.03 \mathrm{eV}, \quad \Delta m_{21}^{2}=\left(7.39_{-0.20}^{+0.21}\right) \times 10^{-5} \mathrm{eV}^{2}, \quad \Delta m_{32}^{2}=\left(2.525_{-0.032}^{+0.033}\right) \times 10^{-3} \mathrm{eV}^{2},
$$

where the neutrino mass squared differences are: $\Delta m_{i j}^{2}=m_{i}^{2}-m_{j}^{2}$. By setting $v_{\text {light }}$ to electroweak scale and $L \sim 8$ or 9 , one obtains light-neutrino masses from the correct ballpark. We will choose these values for our numerical example.

When the FN-charge of the lepton triplet is fixed, the charged lepton mass hierarchy depends only on the FN-charges $q\left(e_{R, i}\right)$ of the right-handed charged leptons as is evident from eq. (4.2). The FN-charges $q\left(e_{R, i}\right)$ are the sole source of charged lepton mass hierarchy, as all the left-handed lepton triplet FN-charges are identical. We choose the right-handed charged lepton charges so that their mass matrix texture becomes:

$$
m^{e} \sim v^{\prime}\left(\begin{array}{ccc}
\epsilon^{9} & \epsilon^{6} & \epsilon^{4} \\
\epsilon^{9} & \epsilon^{6} & \epsilon^{4} \\
\epsilon^{9} & \epsilon^{6} & \epsilon^{4}
\end{array}\right)
$$

As a summary the chosen lepton FN-charges are presented in table 3. The FN-charges of the scalar triplets were presented in table 1.

\subsection{Numerical values for leptons}

We have chosen three benchmark points BP1, BP2 and BP3, presented in table 3. The order-one coefficients introduced in eq. (4.2) and eq. (5.2) are in the interval $|c| \in[0.5,5]$ to retain naturalness of the parameters. We choose different values for $\mathrm{SU}(3)_{L} \times \mathrm{U}(1)_{X^{-}}$ breaking VEVs $u$ and $v_{2}$, the $\mathrm{SU}(2)_{L} \times \mathrm{U}(1)_{Y}$-breaking VEVs $v^{\prime}$ and $v_{1}$, and the FN charges for charged leptons. Below we list the explicit values for the coupling matrices we used.

\section{Benchmark point 1.}

$$
\begin{aligned}
& c_{\eta^{*}}^{N}=\left(\begin{array}{ccc}
0 & 1.4094 & 4.9481 \\
-1.4094 & 0 & 1.5320 \\
-4.9481 & -1.5320 & 0
\end{array}\right) \quad c^{N}=\left(\begin{array}{ccc}
3.8685 & -0.6004 & 2.5618 \\
0.6590 & -0.8619 & 2.6293 \\
-3.3890 & -4.1831 & 4.2469
\end{array}\right) \\
& c^{\prime}=\left(\begin{array}{ccc}
2.5272 & 3.2832 & 2.5651 \\
-1.9815 & 2.3937 & 2.6579 \\
-4.0777 & 4.7926 & -2.0345
\end{array}\right) \quad c^{M}=\left(\begin{array}{ccc}
4.0703 & 2.3618 & 1.3761 \\
2.3618 & -1.1174 & -4.4777 \\
1.3761 & -4.4777 & -2.4131
\end{array}\right) \\
& c^{e}=\left(\begin{array}{ccc}
1.9737 & -3.6284 & -3.0577 \\
-0.5178 & -3.1689 & -2.0976 \\
-0.9467 & 3.1251 & -0.9093
\end{array}\right)
\end{aligned}
$$

\section{Benchmark point 2.}

$$
c_{\eta^{*}}^{N}=\left(\begin{array}{ccc}
0 & 0.5925 & 4.0397 \\
-0.5925 & 0 & 2.1615 \\
-4.0397 & -2.1615 & 0
\end{array}\right) \quad c^{N}=\left(\begin{array}{ccc}
3.7356 & 0.5776 & 2.9485 \\
0.7189 & -1.0852 & 2.5412 \\
-2.7567 & -4.5597 & 4.1934
\end{array}\right)
$$




\begin{tabular}{|c|c|c|c|}
\hline Benchmarks & BP1 & BP2 & BP3 \\
\cline { 1 - 2 }$v^{\prime}(\mathrm{GeV})$ & 237.05 & \multirow{2}{*}{197.5999} & 203.205 \\
\cline { 1 - 2 }$v_{1}(\mathrm{GeV})$ & 100 & & \\
\hline$u(\mathrm{TeV})$ & 48 & 21 & 7 \\
\hline$v_{2}(\mathrm{TeV})$ & 55 & 19 & 7.5 \\
\hline$\Lambda(\mathrm{TeV})$ & 75.8 & 29.5 & 10.7 \\
\hline$q\left(L_{\alpha}^{c}\right)$ & \multicolumn{2}{|c|}{8} & 9 \\
\hline$q\left(e_{R}\right)$ & \multicolumn{2}{|c|}{2} & 1 \\
\hline$q\left(\mu_{R}\right)$ & \multicolumn{2}{|c|}{-1} & -2 \\
\hline$q\left(\tau_{R}\right)$ & \multicolumn{2}{|c|}{-3} & -4 \\
\hline
\end{tabular}

Table 3. The numerical values of vacuum expectation values and FN charge assignments of leptons for our benchmarks.

$$
\begin{aligned}
& c^{\prime N}=\left(\begin{array}{ccc}
1.9602 & 2.6081 & 2.5080 \\
-1.2359 & 2.2310 & 2.2478 \\
-3.8536 & 4.9354 & -1.6207
\end{array}\right) \quad c^{M}=\left(\begin{array}{ccc}
3.5467 & 2.1954 & 1.4849 \\
2.1954 & -1.4909 & -4.3984 \\
1.4849 & -4.3984 & -2.2496
\end{array}\right) \\
& c^{e}=\left(\begin{array}{ccc}
2.0244 & -2.9615 & -3.5841 \\
-1.0302 & -2.3998 & -2.0283 \\
-0.7233 & 3.7798 & -1.9120
\end{array}\right)
\end{aligned}
$$

Benchmark point 3.

$$
\begin{array}{rlrl}
c_{\eta^{*}}^{N} & =\left(\begin{array}{ccc}
0 & -1.2219 & 4.6215 \\
1.2219 & 0 & 1.3745 \\
-4.6215 & -1.3745 & 0
\end{array}\right) & c^{N}=\left(\begin{array}{ccc}
4.1270 & 1.2035 & 1.6042 \\
3.3340 & -1.5694 & 2.8805 \\
-2.2421 & -4.9699 & 2.6798
\end{array}\right) \\
c^{\prime} & =\left(\begin{array}{ccc}
2.95332 & 1.54563 & 2.77676 \\
0.58451 & 3.64608 & 2.22936 \\
-3.42031 & -3.69692 & -2.68750
\end{array}\right) \\
c^{e} & =\left(\begin{array}{ccc}
1.5625 & -3.0524 & -3.7095 \\
-1.2578 & -1.9733 & -1.8557 \\
-1.5279 & 3.9513 & -1.4851
\end{array}\right)
\end{array}
$$

See table 4 for the resulting neutrino masses, mass squared differences and effective strengths of nonunitary and nonstandard interactions, as well as the $V_{\mu}^{ \pm}-W_{\mu}^{ \pm}$mixing angle.

From the figures 1 and 2 it is apparent that next-generation neutrino oscillation experiments measuring $\nu_{\mu}$ disappearance or neutrinoless double beta decay $(0 \nu \beta \beta)$ experiments have a moderate possibility of supporting our model at BP3, since the present experimental limits are only approximately one degree of magnitude weaker. Of our three benchmark points, BP3 has the greatest prospect of being detected in future, since the sterile component of $\nu_{e}$ has a disapprearance effect $\sum_{j=4}^{6}\left|U_{e j}\right|^{2} \sim 10^{-3}$ and similarly the expected $\nu_{\mu}$ disappearance should be $\sum_{j=4}^{6}\left|U_{\mu j}\right|^{2} \sim 10^{-4}$. For BP1 and BP2 the disappearance effect is smaller by a factor of $\mathcal{O}(100)$ and $\mathcal{O}(10)$, respectively. The medium-mass neutrinos 


\begin{tabular}{|c|c|c|c|c|}
\hline Benchmarks & BP1 & BP2 & BP3 & Experimental values \\
\hline Nonunitary strength & \multirow{2}{*}{$\sim 10^{-5}$} & \multirow{2}{*}{$\sim 10^{-4}$} & \multirow{2}{*}{$\sim 10^{-3}$} & \multirow{2}{*}{$\lesssim 0.01$} \\
\hline NSI strength & & & & \\
\hline$m_{1}(\mathrm{meV})$ & 0.0234 & 1.85 & 2.98 & \multirow{2}{*}{$\lesssim 55$} \\
\hline$m_{2}(\mathrm{meV})$ & 8.59 & 8.93 & 9.06 & \\
\hline$m_{3}(\mathrm{meV})$ & 51.2 & 51.1 & 50.8 & $\lesssim 60$ \\
\hline$m_{1}+m_{2}+m_{3}(\mathrm{meV})$ & 59.8 & 61.9 & 62.8 & $<120$ \\
\hline$\Delta m_{21}^{2}\left(10^{-5} \mathrm{eV}^{2}\right)$ & 7.39 & 7.64 & 7.33 & $6.79-8.01$ \\
\hline$\left|\Delta m_{32}^{2}\right|\left(10^{-3} \mathrm{eV}^{2}\right)$ & 2.62 & 2.61 & 2.50 & $2.412-2.625$ \\
\hline$m_{4}(\mathrm{keV})$ & 1.36 & 0.387 & 0.0109 & \multirow{6}{*}{ Unknown } \\
\hline$m_{5}(\mathrm{keV})$ & 4.99 & 2.03 & 0.0206 & \\
\hline$m_{6}(\mathrm{keV})$ & 12.8 & 5.42 & 0.0735 & \\
\hline$m_{7}(\mathrm{TeV})$ & 184 & 69.3 & 11.2 & \\
\hline$m_{8}(\mathrm{TeV})$ & 380 & 129 & 48.2 & \\
\hline$m_{9}(\mathrm{TeV})$ & 523 & 204 & 65.1 & \\
\hline$V_{\mu}^{ \pm}-W_{\mu}^{ \pm}$mixing $|\theta|$ & 0.0010 & 0.0047 & 0.014 & $\lesssim 0.01-0.04$ \\
\hline
\end{tabular}

Table 4. The computed values of neutrino masses, effective neutrino interaction strength and $V_{\mu}^{ \pm}-W_{\mu}^{ \pm}$mixing for our benchmarks.

lie on the $\mathrm{eV}$-scale. For the case on BP3, the lightest sterile neutrino $\nu_{1}^{\prime}$, (mass $m_{4}$ ) it will be able to account the MiniBooNe anomaly [46]. We calculated the active-medium neutrino mixing matrices and have illustrated them at constraint plots. Figure 1 shows the constraints from $0 \nu \beta \beta$ experiments $[47,48]$ and kink searches in single beta decay energy spectra of various unstable radioactive isotopes [48] for BP3. We have also included the expected sensitivity of KATRIN experiment after three-year run. Figure 2 shows the constraints from muon neutrino disappearance experiments [49] and the MiniBooNe anomaly for BP3.

\section{Conclusion}

The FN331-model is based on $\mathrm{SU}(3)_{c} \times \mathrm{SU}(3)_{L} \times \mathrm{U}(1)_{X}$ gauge symmetry and economically incorporates the Froggatt-Nielsen mechanism into it, thus simultaneously explaining the number of fermion families and the mass hierarchy of charged fermions. In this work we extended the FN331-model with three right-handed neutrino singlets. This allowed for tree-level masses for all of the neutrinos, which the original FN331-model was lacking. The neutrino masses and mixings in this model are naturally explained by utilizing a combination of the seesaw and FN mechanisms. Lightest sterile neutrino of our model is a viable candidate for the MiniBooNe oscillation anomaly. The light-neutrino masses acquire additional suppression due to the FN mechanism, allowing the Majorana mass scale to be quite low, around few $\mathrm{TeV}$. This allows for the possible collider searches of the heavy neutrinos in the future colliders. The mixing of the neutrinos, represented by the PMNS-matrix, is also explained without fine-tuning since the FN mechanism can enforce 


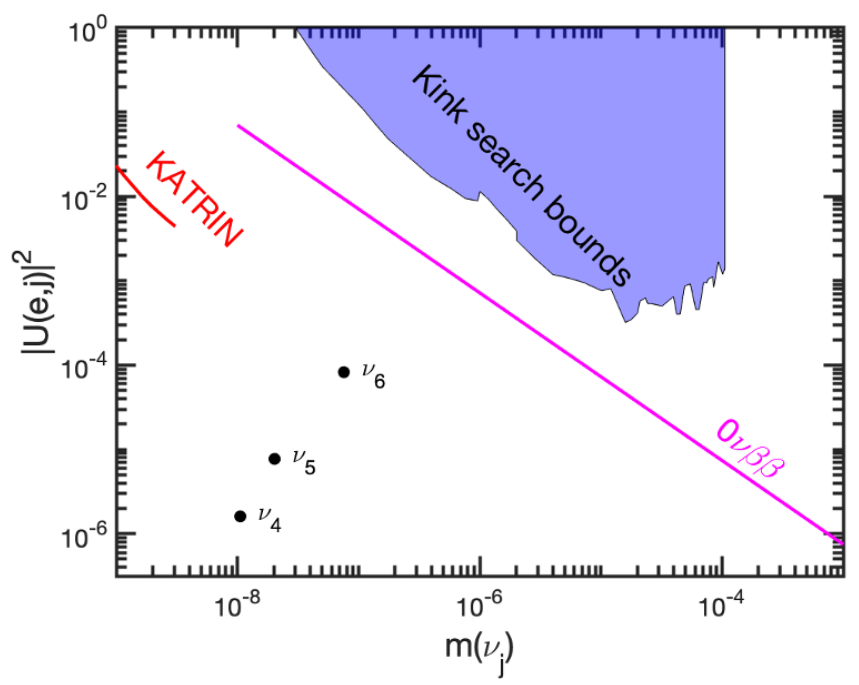

Figure 1. Constraints for the matrix element absolute values squared describing the strength of mixing of electron neutrino and medium-massive neutrinos, $U_{e j}$. Mass is in GeV units. Black dots denote the corresponding values for BP3.

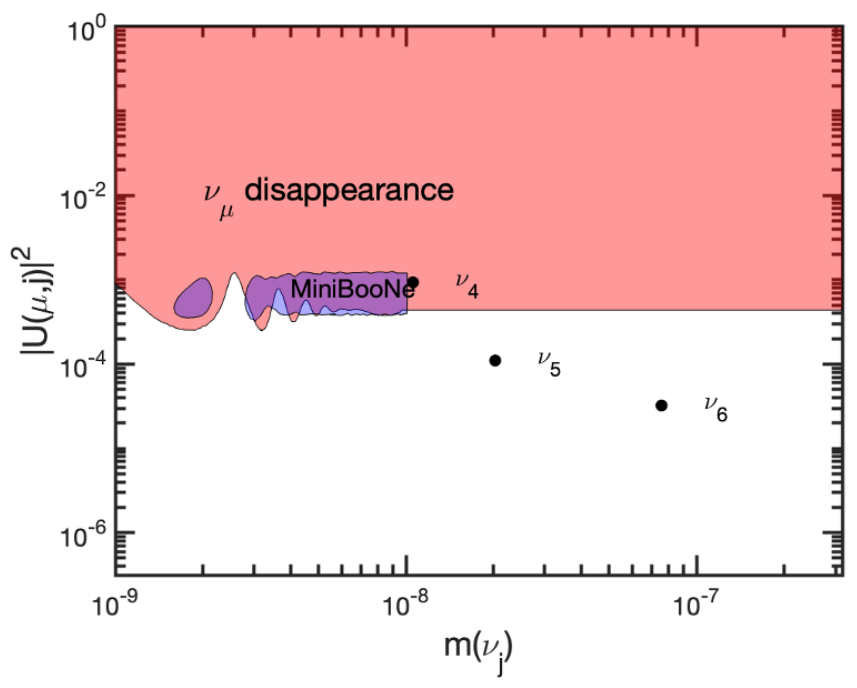

Figure 2. Same as figure 1, but for mixing of muon neutrinos.

the correct texture for the lepton mass matrices. As a summary the model presented here offers an explanation for the whole fermion sector: it explains the number of fermion families and the mass hierarchy of all of the fermions, thus solving the flavour problem while fulfilling all the experimental constraints.

\section{Acknowledgments}

The authors acknowledge the H2020-MSCA-RICE-2014 grant no. 645722 (NonMinimalHiggs). NK is supported by Vilho, Yrjö and Kalle Väisälä Foundation. 


\section{A Scalar mass matrices}

\section{A.1 CP-even scalars}

The CP-even scalar mass term is

$$
\mathcal{L} \supset \frac{1}{2} H^{T} M_{\mathrm{cp}-\mathrm{even}}^{2} H,
$$

where $H^{T}=\left(h_{1}, h_{2}, h_{3}, h_{4}, h_{5}\right)$ and

$$
M_{\text {cp-even }}^{2}=\left(\begin{array}{ccccc}
2 \lambda_{1} v^{\prime 2}+f \frac{v_{1} u}{v^{\prime}} & \lambda_{12} v^{\prime} v_{1}-f u & f v_{2} & \lambda_{12} v^{\prime} v_{2} & \lambda_{13} v^{\prime} u-f v_{1} \\
\lambda_{12} v^{\prime} v_{1}-f u & 2 \lambda_{2} v_{1}^{2}+f \frac{v^{\prime} u}{v_{1}} & \frac{1}{2} \widetilde{\lambda}_{23} v_{2} u+b & 2 \lambda_{2} v_{1} v_{2} & \lambda_{23} v_{1} u-f v^{\prime} \\
f v_{2} & \frac{1}{2} \widetilde{\lambda}_{23} v_{2} u+b & -\frac{1}{2} \widetilde{\lambda}_{23} v_{2}^{2}-b \frac{\left(v_{1}^{2}+v_{2}^{2}\right)}{v_{2} u} & -b \frac{v_{1}}{v_{2}} & \frac{1}{2} \widetilde{\lambda}_{23} v_{1} v_{2} \\
\lambda_{12} v^{\prime} v_{2} & 2 \lambda_{2} v_{1} v_{2} & -b \frac{v_{1}}{v_{2}} & 2 \lambda_{2} v_{2}^{2}-b \frac{u}{v_{2}} & \left(\lambda_{23}+\widetilde{\lambda}_{23}\right) v_{2} u+b \\
\lambda_{13} v^{\prime} u-f v_{1} & \lambda_{23} v_{1} u-f v^{\prime} & \frac{1}{2} \widetilde{\lambda}_{23} v_{1} v_{2} & \left(\lambda_{23}+\widetilde{\lambda}_{23}\right) v_{2} u+b & 2 \lambda_{3} u^{2}+f \frac{v^{\prime} v_{1}}{u}-b \frac{v_{2}}{u}
\end{array}\right) .
$$

\section{A.2 CP-odd scalars}

The CP-odd scalar mass term is

$$
\mathcal{L} \supset \frac{1}{2} A^{T} M_{\text {cp-odd }}^{2} A
$$

where $A^{T}=\left(\xi_{1}, \xi_{2}, \xi_{3}, \xi_{4}, \xi_{5}\right)$ and

$$
M_{\mathrm{cp}-\text { odd }}^{2}=\left(\begin{array}{ccccc}
f \frac{v_{1} u}{v^{\prime}} & f u & 0 & -f v_{2} & f v_{1} \\
f u & f \frac{v^{\prime} u}{v_{1}} & 0 & \frac{1}{2} \widetilde{\lambda}_{23} v_{2} u+b & f v^{\prime} \\
0 & 0 & -b \frac{u}{v_{2}} & b \frac{v_{1}}{v_{2}} & b \\
-f v_{2} & \frac{1}{2} \widetilde{\lambda}_{23} v_{2} u+b & b \frac{v_{1}}{v_{2}} & -\frac{1}{2} \widetilde{\lambda}_{23} v_{2}^{2}-b \frac{\left(v_{1}^{2}+v_{2}^{2}\right)}{v_{2} u} & \frac{1}{2} \lambda_{23} v_{1} v_{2} \\
f v_{1} & f v^{\prime} & b & \frac{1}{2} \lambda_{23} v_{1} v_{2} & f \frac{v^{\prime} v_{1}}{u}-b \frac{v_{2}}{u}
\end{array}\right) .
$$

\section{A.3 Charged scalars}

The charged scalar mass term is

$$
\mathcal{L} \supset C^{T} M_{\text {charged scalar }}^{2} C,
$$

where $C^{T}=\left(\eta^{+}, \eta^{+}, \rho^{+}, \chi^{+}\right)$and

$$
M_{\text {charged scalar }}^{2}=\left(\begin{array}{cccc}
f \frac{v_{1} u}{v^{\prime}}+\frac{1}{2} \widetilde{\lambda}_{12} v_{2}^{2}+\frac{1}{2} \widetilde{\lambda}_{13} u^{2} & \frac{1}{2} \widetilde{\lambda}_{12} v_{1} v_{2} & \frac{1}{2} \widetilde{\lambda}_{12} v^{\prime} v_{2} & \frac{1}{2} \widetilde{\lambda}_{13} v^{\prime} u+f v_{1} \\
\frac{1}{2} \widetilde{\lambda}_{12} v_{1} v_{2} & f \frac{v_{1} u}{v^{\prime}}+\frac{1}{2} \widetilde{\lambda}_{12} v_{1}^{2} & \frac{1}{2} \widetilde{\lambda}_{12} v^{\prime} v_{1}+f u & -f v_{2} \\
\frac{1}{2} \widetilde{\lambda}_{12} v^{\prime} v_{2} & \frac{1}{2} \widetilde{\lambda}_{12} v^{\prime} v_{1}+f u & f \frac{v^{\prime} u}{v_{1}}+\frac{1}{2} \widetilde{\lambda}_{12} v^{\prime 2} & \frac{1}{2} \widetilde{\lambda}_{23} v_{2} u+b \\
\frac{1}{2} \widetilde{\lambda}_{13} v^{\prime} u+f v_{1} & -f v_{2} & \frac{1}{2} \widetilde{\lambda}_{23} v_{2} u+b & f \frac{v^{\prime} v_{1}}{u}-\frac{1}{2} \widetilde{\lambda}_{23} v_{2}^{2}+\frac{1}{2} \widetilde{\lambda}_{13} v^{\prime 2}-b \frac{v_{2}}{u}
\end{array}\right) .
$$

\section{B Neutral gauge boson masses}

There are five neutral gauge bosons: $W_{3 \mu}, W_{\mu}, B_{\mu}, W_{4 \mu}$ and $W_{5 \mu}$. The imaginary part of $X_{\mu}^{\prime 0}$ decouples from the other neutral gauge bosons and acquires a mass:

$$
M_{W_{5}}^{2}=\frac{g_{3}^{2}}{4}\left(v_{1}^{2}+v_{2}^{2}+u^{2}\right) .
$$


The rest of the neutral gauge bosons mix,

$$
\mathcal{L} \supset \frac{1}{2} X^{T} M_{\text {neutral }}^{2} X,
$$

where the basis is $X^{T}=\left(W_{3 \mu}, W_{8 \mu}, B_{\mu}, W_{4 \mu}\right)$ and

$$
M_{\text {neutral }}^{2}=\frac{g_{3}^{2}}{4}\left(\begin{array}{cccc}
v^{\prime 2}+v_{1}^{2} & \frac{v_{1}^{2}-v^{\prime 2}}{\sqrt{3}} & -\frac{2 g_{x}}{3 g_{3}}\left(v_{1}^{2}+2 v^{\prime 2}\right) & v_{1} v_{2} \\
\frac{v_{1}^{2}-v^{\prime 2}}{\sqrt{3}} & \frac{\left(v^{\prime 2}+v_{1}^{2}+4\left(v_{2}^{2}+u_{2}^{2}\right)\right)}{3} & \frac{\left(-v_{1}^{2}+2\left(v_{2}^{2}+u_{2}^{2}+v^{\prime 2}\right)\right)}{\left(\frac{3 \sqrt{3} g_{3}}{2 g_{x}}\right)} & -\frac{v_{1} v_{2}}{\sqrt{3}} \\
-\frac{2 g_{x}}{3 g_{3}}\left(v_{1}^{2}+2 v^{\prime 2}\right) & \frac{\left(-v_{1}^{2}+2\left(v_{2}^{2}+u_{2}^{2}+v^{\prime 2}\right)\right)}{\left(\frac{3 \sqrt{3} g_{3}}{2 g_{x}}\right)} & \frac{\left(v_{1}^{2}+v_{2}^{2}+u_{2}^{2}+4 v^{\prime 2}\right)}{\left(\frac{9 g_{3}^{2}}{4 g_{x}^{2}}\right)} & -\frac{4 g_{x}}{3 g_{3}}\left(v_{1} v_{2}\right) \\
v_{1} v_{2} & -\frac{v_{1} v_{2}}{\sqrt{3}} & -\frac{4 g_{x}}{3 g_{3}}\left(v_{1} v_{2}\right) & v_{1}^{2}+v_{2}^{2}+u_{2}^{2}
\end{array}\right) .
$$

The eigenvalues of this matrix can be solved analytically and they are

$$
\begin{aligned}
m_{\gamma}^{2} & =0, \\
m_{\widetilde{W}_{4}}^{2} & =\frac{g_{3}^{2}}{4}\left(u^{2}+v_{2}^{2}+v_{1}^{2}\right), \\
m_{Z}^{2} & =\frac{g_{3}^{2}}{4}\left(\frac{3 g_{3}^{2}+4 g_{x}^{2}}{3 g_{3}^{2}+g_{x}^{2}}\right)\left(v^{\prime 2}+\frac{v_{1}^{2} u^{2}}{v_{2}^{2}+u^{2}}\right)+\mathcal{O}\left(\frac{v_{\text {light }}^{2}}{v_{\text {heavy }}^{2}}\right), \\
m_{Z^{\prime}}^{2} & =\frac{3 g_{3}^{2}+g_{x}^{2}}{9}\left(v_{2}^{2}+u^{2}\right)+\mathcal{O}\left(\frac{v_{\text {light }}^{2}}{v_{\text {heavy }}^{2}}\right) .
\end{aligned}
$$

One notices that one of the eigenvalues is exactly the same as that of the imaginary part of the non-hermitian gauge boson. We can therefore identify the combination

$$
X_{\mu}^{0}=\frac{1}{\sqrt{2}}\left(W_{4 \mu}-i W_{5 \mu}\right)
$$

as the physical neutral non-hermitean gauge boson.

Open Access. This article is distributed under the terms of the Creative Commons Attribution License (CC-BY 4.0), which permits any use, distribution and reproduction in any medium, provided the original author(s) and source are credited.

\section{References}

[1] Particle Data Group collaboration, Review of particle physics, Phys. Rev. D 98 (2018) 030001 [INSPIRE].

[2] G.S. Abrams et al., Measurements of $Z$ boson resonance parameters in $e^{+} e^{-}$annihilation, Phys. Rev. Lett. 63 (1989) 2173 [INSPIRE].

[3] L3 collaboration, Determination of the number of light neutrino species, Phys. Lett. B 292 (1992) 463 [INSPIRE].

[4] OPAL collaboration, Measurement of single photon production in $e^{+} e^{-}$collisions near the Z0 resonance, Z. Phys. C 65 (1995) 47 [INSPIRE]. 
[5] ALEPH collaboration, $A$ direct measurement of the invisible width of the $Z$ from single photon counting, Phys. Lett. B 313 (1993) 520 [INSPIRE].

[6] L3 collaboration, A Direct determination of the number of light neutrino families from $e^{+} e^{-} \rightarrow \nu \bar{\nu} \gamma$ at LEP, Phys. Lett. B 275 (1992) 209 [Erratum ibid. B 277 (1992) 530] [INSPIRE].

[7] OPAL collaboration, $A$ direct measurement of the $Z 0$ invisible width by single photon counting, Z. Phys. C 50 (1991) 373 [INSPIRE].

[8] F. Pisano and V. Pleitez, An SU(3) $\times \mathrm{U}(1)$ model for electroweak interactions, Phys. Rev. D 46 (1992) 410 [hep-ph/9206242] [INSPIRE].

[9] P.H. Frampton, Chiral dilepton model and the flavor question, Phys. Rev. Lett. 69 (1992) 2889 [INSPIRE].

[10] R. Foot, O.F. Hernandez, F. Pisano and V. Pleitez, Lepton masses in an $\mathrm{SU}(3)_{L} \times \mathrm{U}(1)_{N}$ gauge model, Phys. Rev. D 47 (1993) 4158 [hep-ph/9207264] [INSPIRE].

[11] M.D. Tonasse, The scalar sector of 3-3-1 models, Phys. Lett. B 381 (1996) 191 [hep-ph/9605230] [INSPIRE].

[12] T.A. Nguyen, N.A. Ky and H.N. Long, The Higgs sector of the minimal 3-3-1 model revisited, Int. J. Mod. Phys. A 15 (2000) 283 [hep-ph/9810273] [INSPIRE].

[13] H. Georgi and A. Pais, Generalization of Gim: horizontal and vertical flavor mixing, Phys. Rev. D 19 (1979) 2746 [INSPIRE].

[14] M. Singer, J.W.F. Valle and J. Schechter, Canonical neutral current predictions from the weak electromagnetic gauge group $\mathrm{SU}(3) \times \mathrm{U}(1)$, Phys. Rev. D 22 (1980) 738 [INSPIRE].

[15] J.W.F. Valle and M. Singer, Lepton number violation with quasi Dirac neutrinos, Phys. Rev. D 28 (1983) 540 [INSPIRE].

[16] J.C. Montero, F. Pisano and V. Pleitez, Neutral currents and GIM mechanism in $\mathrm{SU}(3)_{L} \times \mathrm{U}(1)_{N}$ models for electroweak interactions, Phys. Rev. D 47 (1993) 2918 [hep-ph/9212271] [INSPIRE].

[17] R. Foot, H.N. Long and T.A. Tran, $\mathrm{SU}(3)_{L} \otimes U(1)_{N}$ and $\mathrm{SU}(4)_{L} \otimes U(1)_{N}$ gauge models with right-handed neutrinos, Phys. Rev. D 50 (1994) R34 [hep-ph/9402243] [InSPIRE].

[18] H.N. Long, The 331 model with right handed neutrinos, Phys. Rev. D 53 (1996) 437 [hep-ph/9504274] [INSPIRE].

[19] H.N. Long, $\mathrm{SU}(3)_{L} \times \mathrm{U}(1)_{N}$ model for right-handed neutrino neutral currents, Phys. Rev. D 54 (1996) 4691 [hep-ph/9607439] [INSPIRE].

[20] V. Pleitez, New fermions and a vector-like third generation in $\mathrm{SU}(3)(C) \times \mathrm{SU}(3)(L) \times \mathrm{U}(1)(N)$ models, Phys. Rev. D 53 (1996) $514[$ hep-ph/9412304] [INSPIRE].

[21] H.N. Long, Scalar sector of the 3-3-1 model with three Higgs triplets, Mod. Phys. Lett. A 13 (1998) 1865 [hep-ph/9711204] [INSPIRE].

[22] P.V. Dong, T.P. Nguyen and D.V. Soa, 3-3-1 model with inert scalar triplet, Phys. Rev. D 88 (2013) 095014 [arXiv: 1308.4097] [INSPIRE].

[23] L.T. Hue and L.D. Ninh, The simplest 3-3-1 model, Mod. Phys. Lett. A 31 (2016) 1650062 [arXiv: 1510.00302] [INSPIRE].

[24] C.D. Froggatt and H.B. Nielsen, Hierarchy of quark masses, Cabibbo angles and CP-violation, Nucl. Phys. B 147 (1979) 277 [inSPIRE]. 
[25] K. Huitu and N. Koivunen, Froggatt-Nielsen mechanism in a model with $\mathrm{SU}(3)_{c} \times \mathrm{SU}(3)_{L} \times \mathrm{U}(1)_{X}$ gauge group, Phys. Rev. D 98 (2018) 011701 [arXiv:1706.09463] [INSPIRE].

[26] K. Huitu and N. Koivunen, Suppression of scalar mediated FCNCs in a $\mathrm{SU}(3)_{c} \times \mathrm{SU}(3)_{L} \times \mathrm{U}(1)_{X}$-model, JHEP 10 (2019) 065 [arXiv: 1905. 05278] [InSPIRE].

[27] C. Salazar, R.H. Benavides, W.A. Ponce and E. Rojas, LHC constraints on 3-3-1 models, JHEP 07 (2015) 096 [arXiv: 1503.03519] [INSPIRE].

[28] M.E. Catano, R. Martinez and F. Ochoa, Neutrino masses in a 3-3-1 model with right-handed neutrinos without doubly charged Higgs bosons via inverse and double seesaw mechanisms, Phys. Rev. D 86 (2012) 073015 [arXiv:1206.1966] [InSPIRE].

[29] P. Minkowski, $\mu \rightarrow$ er at a rate of one out of $10^{9}$ muon decays?, Phys. Lett. B 67 (1977) 421.

[30] T. Yanagida, Horizontal gauge symmetry and masses of neutrinos, Conf. Proc. C 7902131 (1979) 95 [INSPIRE].

[31] M. Gell-Mann, P. Ramond and R. Slansky, Complex spinors and unified theories, Conf. Proc. C 790927 (1979) 315 [arXiv:1306.4669] [INSPIRE].

[32] R.N. Mohapatra and G. Senjanović, Neutrino mass and spontaneous parity nonconservation, Phys. Rev. Lett. 44 (1980) 912 [INSPIRE].

[33] J. Schechter and J.W.F. Valle, Neutrino masses in $\mathrm{SU}(2) \times \mathrm{U}(1)$ theories, Phys. Rev. D 22 (1980) 2227 [INSPIRE].

[34] S.L. Glashow, The future of elementary particle physics, NATO Sci. Ser. B 61 (1980) 687.

[35] I. Esteban et al., Global analysis of three-flavour neutrino oscillations: synergies and tensions in the determination of $\theta_{23}, \delta_{\mathrm{CP}}$ and the mass ordering, JHEP 01 (2019) 106 [arXiv: 1811.05487] [INSPIRE].

[36] NuFIT 4.0, www.nu-fit.org (2018).

[37] M. Blennow et al., Non-unitarity, sterile neutrinos and non-standard neutrino interactions, JHEP 04 (2017) 153 [arXiv: 1609.08637] [INSPIRE].

[38] F.J. Escrihuela et al., On the description of nonunitary neutrino mixing, Phys. Rev. D 92 (2015) 053009 [Erratum ibid. D 93 (2016) 119905] [arXiv: 1503.08879] [INSPIRE].

[39] S. Antusch et al., Unitarity of the leptonic mixing matrix, JHEP 10 (2006) 084 [hep-ph/0607020] [INSPIRE].

[40] S. Antusch, P. Huber, S.F. King and T. Schwetz, Neutrino mixing sum rules and oscillation experiments, JHEP 04 (2007) 060 [hep-ph/0702286] [INSPIRE].

[41] S.F. King, Predicting neutrino parameters from $\mathrm{SO}(3)$ family symmetry and quark-lepton unification, JHEP 08 (2005) 105 [hep-ph/0506297] [INSPIRE].

[42] I. Masina, A maximal atmospheric mixing from a maximal CP-violating phase, Phys. Lett. B 633 (2006) 134 [hep-ph/0508031] [INSPIRE].

[43] C. Kraus et al., Final results from phase II of the Mainz neutrino mass search in tritium beta decay, Eur. Phys. J. C 40 (2005) 447 [hep-ex/0412056] [INSPIRE].

[44] KamLAND-Zen collaboration, Limit on neutrinoless $\beta \beta$ decay of ${ }^{136}$ Xe from the first phase of KamLAND-Zen and comparison with the positive claim in ${ }^{76} G e$, Phys. Rev. Lett. 110 (2013) 062502 [arXiv:1211.3863] [INSPIRE].

[45] Planck collaboration, Planck 2018 results. VI. Cosmological parameters, arXiv: 1807.06209 [INSPIRE]. 
[46] MiniBoonE collaboration, Significant excess of electronlike events in the MiniBooNE short-baseline neutrino experiment, Phys. Rev. Lett. 121 (2018) 221801 [arXiv:1805.12028] [INSPIRE].

[47] P. Benes, A. Faessler, F. Simkovic and S. Kovalenko, Sterile neutrinos in neutrinoless double beta decay, Phys. Rev. D 71 (2005) 077901 [hep-ph/0501295] [INSPIRE].

[48] A. Atre, T. Han, S. Pascoli and B. Zhang, The search for heavy Majorana neutrinos, JHEP 05 (2009) 030 [arXiv:0901.3589] [INSPIRE].

[49] KARMEN collaboration, Upper limits for neutrino oscillations muon-anti-neutrino $\rightarrow$ electron-anti-neutrino from muon decay at rest, Phys. Rev. D 65 (2002) 112001 [hep-ex/0203021] [INSPIRE].

[50] R.M. Fonseca and M. Hirsch, A flipped 331 model, JHEP 08 (2016) 003 [arXiv:1606.01109] [INSPIRE].

[51] A.E. Cárcamo Hernández, S. Kovalenko, H.N. Long and I. Schmidt, A variant of 3-3-1 model for the generation of the SM fermion mass and mixing pattern, JHEP 07 (2018) 144 [arXiv: 1705.09169] [INSPIRE].

[52] J.T. Liu and D. Ng, Lepton flavor changing processes and CP-violation in the 331 model, Phys. Rev. D 50 (1994) 548 [hep-ph/9401228] [INSPIRE].

[53] P.V. Dong and H.N. Long, Neutrino masses and lepton flavor violation in the 3-3-1 model with right-handed neutrinos, Phys. Rev. D 77 (2008) 057302 [arXiv:0801.4196] [INSPIRE].

[54] S.M. Boucenna, J.W.F. Valle and A. Vicente, Predicting charged lepton flavor violation from 3-3-1 gauge symmetry, Phys. Rev. D 92 (2015) 053001 [arXiv:1502.07546] [INSPIRE].

[55] A.E. Cárcamo Hernández, Y. Hidalgo Velásquez and N.A. Pérez-Julve, A 3-3-1 model with low scale seesaw mechanisms, Eur. Phys. J. C 79 (2019) 828 [arXiv:1905.02323] [INSPIRE]. 